\title{
INVOLUTORY AUTOMORPHISMS OF OPERATOR ALGEBRAS
}

BY

\author{
E. B. DAVIES( $\left.{ }^{(}\right)$
}

\begin{abstract}
We develop the mathematical machinery necessary in order to describe systematically the commutation and anticommutation relations of the field algebras of an algebraic quantum field theory of the fermion type. In this context it is possible to construct a skew tensor product of two von Neumann algebras and completely describe its type in terms of the types of the constituent algebras. Mathematically the paper is a study of involutory automorphisms of $W^{*}$-algebras, of particular importance to quantum field theory being the outer involutory automorphisms of the type III factors. It is shown that each of the hyperfinite type III factors studied by Powers has at least two outer involutory automorphisms not conjugate under the group of all automorphisms of the factor.
\end{abstract}

1. Introduction. In the application of $C^{*}$-algebras to the study of quantum fields of the fermion type complications arise because of the fact that both commutation and anticommutation relations need to be treated in a systematic manner. While the algebras of local observables form a system of local algebras in the sense of Haag and Kastler [10], this is not true of the full field algebras. In this paper we develop the mathematical machinery necessary for a fuller description of the field algebras. The main novelty is the definition of a $W_{2}^{*}$-algebra as a $W^{*}$-algebra $\mathscr{A}$ provided with a *-automorphism $\theta$ of $\mathscr{A}$ satisfying $\theta^{2}=1$. The algebra $\mathscr{A}=\mathscr{A}_{0}+\mathscr{A}_{1}$, where $\mathscr{A}_{0}$ is the subalgebra of elements $A$ such that $\theta A=A$ and $\mathscr{A}_{1}$ is the subspace of elements $A$ such that $\theta A=-A$.

In $\S 2$ we present the basic theory of a $W_{2}^{*}$-algebra acting on a Hilbert space. We define the opposed $W_{2}^{*}$-algebra, a natural generalisation of the commutant for $W^{*}$-algebras, and prove that the second opposed $W_{2}^{*}$-algebra is equal to the original $W_{2}^{*}$-algebra. We identify the centre of a $W_{2}^{*}$-algebra and see that, as in the case of $W^{*}$-algebras, a general $W_{2}^{*}$-algebra can be studied by first analysing the $W_{2}^{*}$-factors and then using the usual techniques of integral decomposition theory. Independently of their numerical type the $W_{2}^{*}$-factors can be classified into three types which are described as follows. A Type A $W_{2}^{*}$-factor consists of a $W^{*}$-factor together with an inner involutory automorphism $\theta$. A Type B $W_{2}^{*}$-factor is a direct product of two isomorphic $W^{*}$-factors, and the involutory automorphism

Received by the editors April 22, 1970.

AMS 1969 subject classifications. Primary 4665.

Key words and phrases. $C^{*}$-algebras, involutory automorphisms, anticommutation relations, hyperfinite von Neumann algebras.

(1) Work supported by A.F.O.S.R. contract no. F44620-67-C-0029 while the author was on leave at the Massachusetts Institute of Technology.

Copyright (C) 1971, American Mathematical Society 
$\theta$ interchanges elements of the two factors. A Type $\mathrm{C} W_{2}^{*}$-factor consists of a $W^{*}$-factor with an outer involutory automorphism $\theta$; alternatively a $W_{2}^{*}$-algebra $\mathscr{A}, \theta$ is a Type $\mathrm{C} W_{2}^{*}$-factor if and only if both $\mathscr{A}$ and $\mathscr{A}_{0}$ are $W^{*}$-factors.

In $\S 3$ we study tensor products of $W_{2}^{*}$-algebras. There are two ways of defining a tensor product, the more interesting skew tensor product being the natural infinite-dimensional, topological generalisation of the tensor product of two associative linear algebras graded over the additive group $Z_{2}$. The skew product $\mathscr{A} \otimes_{2} \mathscr{B}$ contains $\mathscr{A}$ and $\mathscr{B}$ as $W_{2}^{*}$-subalgebras in such a way that $A B=(-)^{i j} B A$ for all $A \in \mathscr{A}_{i}$ and $B \in \mathscr{B}_{j}$. We show that the skew product of two $W_{2}^{*}$-factors is again a $W_{2}^{*}$-factor and that its type, both in the numerical sense and in the sense outlined above, can be completely determined from the types of the constituent algebras. We outline two examples from algebraic quantum field theory to show how the skew tensor product arises naturally there. The section is concluded by explicit computations of the finite-dimensional cases for later use. The interested reader will be able to check that the finite-dimensional complex Clifford algebras are all $W_{2}^{*}$-factors in a natural sense [1], but there are other finite-dimensional $W_{2}^{*}$ factors in addition.

As preparation for more detailed analysis of the Type C $W_{2}^{*}$-factors we develop in $\S 4$ the necessary theory of representations of $C_{2}^{*}$-algebras. All of the usual ideas about states, cyclic representations, factor representations, etc., have their analogues for $C_{2}^{*}$-algebras. Irreducible representations in our sense correspond to extremal invariant states and are divided into those of Types A and B. For most of the section we concentrate on the class of U.H.F. $C_{2}^{*}$-algebras, which have proved important for classifying representations of the canonical anticommutation relations-in this connection see [17]. For these we obtain a necessary and sufficient condition for a state to be a Type $\mathrm{C}$ factor state by extending arguments due to Powers [13]. It turns out that a product state of a U.H.F. $C_{2}^{*}$-algebra is almost always of Type C. In other words, an involutory automorphism of a hyperfinite factor is typically outer.

In $\S 5$ we consider the major mathematical problem of the theory, the classification up to algebraic isomorphism of all $W_{2}^{*}$-factors. This can be broken up into two simpler problems. The first is the classification of all $W^{*}$-factors, and still remains open thirty years after it was first raised. The other is the classification in a given $W^{*}$-factor of all the conjugacy classes of the involutory automorphisms in the group of all automorphisms. It is an immediate consequence of the theorems on the comparison of projections that any two inner involutory automorphisms of a type III factor are conjugate, and one might hope that the same is true of any two outer involutory automorphisms in a type III factor. We show that this is not so by constructing two nonconjugate outer involutory automorphisms of each of the hyperfinite type III factors studied by Powers [13]. In fact we construct an infinite number of such automorphisms, but all except one turn out to be conjugate to each other. Our results pose more questions than they solve, but show how 
little is known about the automorphism group of any type III factor, and suggest that the second problem above may be as difficult as the first problem has proven to be.

In $\S 6$ we show how $W_{2}^{*}$-algebras are related to the theory of group representations. To do this we introduce the idea of an epigroup over a ring $R$, a concept which appears to be new even at the algebraic level. Representations of 2-epigroups are similar to spinor representations or ordinary locally compact groups.

The author would like to thank Professor I. Segal for some valuable comments on this work.

2. Structure of $W_{2}^{*}$-algebras of operators. Let $\mathscr{H}$ be a Hilbert space and $\mathscr{S}$ a set of bounded operators on $\mathscr{H}$ such that $A \in \mathscr{S}$ implies $A^{*} \in \mathscr{S}$. Let $\mathscr{A}_{1}$ be the closure in the weak operator topology of the odd polynomials in elements of $\mathscr{S}$, and let $\mathscr{A}_{0}$ be the weak closure of the even polynomials. Then $\mathscr{A}_{0}$ is a von Neumann algebra and $\mathscr{A}_{0} \cdot \mathscr{A}_{1} \subseteq \mathscr{A}_{1}, \mathscr{A}_{1} \cdot \mathscr{A}_{0} \subseteq \mathscr{A}_{1}, \mathscr{A}_{1} \cdot \mathscr{A}_{1} \subseteq \mathscr{A}_{0}$.

Proposition 2.1. If $\mathscr{A}=\mathscr{A}_{0}+\mathscr{A}_{1}$ then $\mathscr{A}$ is a von Neumann algebra. $\mathscr{A}_{0} \cap \mathscr{A}_{1}$ is $a$ weakly closed ideal whose identity element we denote by $(1-P)$ where $P$ is a central projection of $\mathscr{A}$. The von Neumann algebra $P \mathscr{A}$ has a unique involutory *-automorphism $\theta$ such that

$$
P \mathscr{A}_{0}=\{A \in P \mathscr{A}: \theta A=A\} \quad \text { and } P \mathscr{A}_{1}=\{A \in P \mathscr{A}: \theta A=-A\} .
$$

Proof. Let $\mathscr{S}^{\prime}$ be the set of operators on $\mathscr{H}^{\prime}=\mathscr{H} \oplus \mathscr{H}$ of the form $(A,-A)$, where $A \in \mathscr{S}$ and we denote by $(A, B)$ the operator $(\xi, \eta) \rightarrow(A \xi, B \eta)$, and let $\mathscr{A}_{0}^{\prime}$ and $\mathscr{A}_{1}^{\prime}$ be constructed from $\mathscr{S}^{\prime}$ as above. Then

$$
\mathscr{A}_{0}^{\prime}=\left\{(A, A): A \in \mathscr{A}_{0}\right\} \quad \text { and } \quad \mathscr{A}_{1}^{\prime}=\left\{(A,-A): A \in \mathscr{A}_{1}\right\}
$$

and, if $\mathscr{A}^{\prime}=\mathscr{A}_{0}^{\prime}+\mathscr{A}_{1}^{\prime}$, then $\mathscr{A}^{\prime}$ is a von Neumann algebra. If $P^{\prime}$ is the projection $P^{\prime}(\xi, \eta)=(\xi, 0)$ then $P^{\prime}$ lies in the commutant of $\mathscr{A}^{\prime}$ and by [6] $P^{\prime} \mathscr{A}^{\prime} P^{\prime}$ is a von Neumann algebra. But $P^{\prime} \mathscr{A}^{\prime} P^{\prime}=P^{\prime} \mathscr{A}_{0}^{\prime} P^{\prime}+P^{\prime} \mathscr{A}_{1}^{\prime} P^{\prime}$ can be identified with $\mathscr{A}=\mathscr{A}_{0}+\mathscr{A}_{1}$ so $\mathscr{A}$ is weakly closed. The set $\mathscr{I}=\mathscr{A}_{0} \cap \mathscr{A}_{1}$ is weakly closed and is stable under multiplication on both sides by elements of $\mathscr{A}_{0}$ or $\mathscr{A}_{1}$ and so is a twosided ideal. As $P \mathscr{A}_{0} \cap P \mathscr{A}_{1}=0, P \mathscr{A}$ is the direct sum of $P \mathscr{A}_{0}$ and $P \mathscr{A}_{1}$. If $X \in P \mathscr{A}$ we can write $X=X_{0}+X_{1}$ uniquely where $X_{0} \in P \mathscr{A}_{0}$ and $X_{1} \in P \mathscr{A}_{1}$ and we define $\theta X=X_{0}-X_{1}$. It is immediate that $\theta$ is an involutory *-automorphism and that the other statements of the proposition hold.

If $\mathscr{S}$ is any set of operators on a Hilbert space $\mathscr{H}$ we define

$$
\mathscr{S}^{c}=\{B \in \mathscr{L}(\mathscr{H}): A B=B A \text { for all } A \in \mathscr{S}\}
$$

and

$$
\mathscr{S}^{a}=\{B \in \mathscr{L}(\mathscr{H}): A B=-B A \text { for all } A \in \mathscr{S}\}
$$

We define a symmetry $R$ on $\mathscr{H}$ as a bounded operator $R$ such that $R \neq I, R^{2}=I$, $R^{*}=R$. If $R$ is a symmetry and $A \in \mathscr{S}$ implies $A^{*} \in \mathscr{S}$ and $A R=-R A$, then 
$A R=-R A$ for all $A \in \mathscr{A}_{1}$ and $A R=R A$ for all $A \in \mathscr{A}_{0}$. Therefore in this case $\mathscr{A}_{0} \cap \mathscr{A}_{1}=0, P=I$, and the ${ }^{*}$-automorphism $\theta$ is given by $\theta A=R A R$ for all $A \in \mathscr{A}$. We call such a $*$-automorphism $\theta$ spatial.

If $\mathscr{A}$ is a von Neumann algebra with an involutory *automorphism $\theta$ then $\theta$ is isometric and ultraweakly bicontinuous by [6], so $\mathscr{A}_{1}=\{A \in \mathscr{A}: \theta A=-A\}$ and $\mathscr{A}_{0}=\{A \in \mathscr{A}: \theta A=A\}$ are weakly closed. If $\mathscr{I}$ is the von Neumann algebra generated by $\mathscr{A}_{1}$ then $\mathscr{I}$ is a weakly closed $\theta$-stable ideal in $\mathscr{A}$ whose identity element $P$ must satisfy $\theta P=P$ and so must lie in $\mathscr{A}_{0} \cap \mathscr{A}^{c}$. Then $\theta$ restricted to $(1-P) \mathscr{A}$ is the identity map. We now define a $W_{2}^{*}$-algebra as a von Neumann algebra with an involutory *-automorphism $\theta$ such that $\mathscr{A}$ is generated by $\mathscr{A}_{1}$.

Proposition 2.2. If $\mathscr{A}, \theta$ is a $W_{2}^{*}$-algebra of operators on $\mathscr{H}$ there is a unique $W_{2}^{*}$-algebra $\mathscr{B}, \varphi$ such that $\mathscr{B}=\mathscr{A}_{0}^{c}, \mathscr{B}_{0}=\mathscr{A}^{c}, \mathscr{B}_{1}=\mathscr{A}_{1}^{a}$. If this is called the opposed algebra then the second opposed algebra of $\mathscr{A}, \theta$ is $\mathscr{A}, \theta$ itself.

Proof. Let us first consider the case where $\theta$ is spatial induced by the symmetry $R$ of $\mathscr{H}$. Defining $\mathscr{B}_{0}=\mathscr{A}^{c}$ and $\mathscr{B}_{1}=\mathscr{A}_{1}^{a}$ it follows from $\mathscr{B}_{0} \cdot \mathscr{B}_{0} \subseteq \mathscr{B}_{0}, \mathscr{B}_{0} \cdot \mathscr{B}_{1} \subseteq \mathscr{B}_{1}$, $\mathscr{B}_{1} \cdot \mathscr{B}_{0} \subseteq \mathscr{B}_{1}, \mathscr{B}_{1} \cdot \mathscr{B}_{1} \subseteq \mathscr{B}_{0}$ that $\mathscr{B}=\mathscr{B}_{0}+\mathscr{B}_{1}$ is a von Neumann algebra satisfying $\mathscr{A}^{c} \subseteq \mathscr{B} \subseteq \mathscr{A}_{0}^{c}$. Therefore $\mathscr{A} \supseteq \mathscr{B}^{c} \supseteq \mathscr{A}_{0}$ and if $\mathscr{B}^{c} \neq \mathscr{A}_{0}$ there exists a nonzero $A \in \mathscr{B}^{c} \cap \mathscr{A}_{1}$; but this is impossible since then $R \in \mathscr{B} \cap \mathscr{A}_{1}^{a}$ would have to commute and anticommute with $A$. Therefore $\mathscr{B}^{c}=\mathscr{A}_{0}$ and $\mathscr{A}_{0}^{c}=\mathscr{B}$.

In the general case we define $\mathscr{H}^{\prime}, \mathscr{A}_{0}^{\prime}, \mathscr{A}_{1}^{\prime}, \mathscr{A}^{\prime}, P^{\prime}$ as in the proof of Proposition 2.1. If $\mathscr{B}^{\prime}=\left(\mathscr{A}_{0}^{\prime}\right)^{c}, \mathscr{B}_{0}^{\prime}=\left(\mathscr{A}^{\prime}\right)^{c}, \mathscr{B}_{1}^{\prime}=\left(\mathscr{A}_{1}^{\prime}\right)^{a}$ we have shown that $\mathscr{B}^{\prime}=\mathscr{B}_{0}^{\prime}+\mathscr{B}_{1}^{\prime}$ and $P^{\prime} \in \mathscr{B}_{0}^{\prime}$. Now defining $\mathscr{B}=P^{\prime} \mathscr{B}^{\prime} P^{\prime}, \mathscr{B}_{0}=P^{\prime} \mathscr{B}_{0}^{\prime} P^{\prime}, \mathscr{B}_{1}=P^{\prime} \mathscr{B}_{1}^{\prime} P^{\prime}$ it is shown in [6] that we can identify $\mathscr{B}_{0}=\mathscr{A}^{c}, \mathscr{B}=\mathscr{A}^{c}$ and that $\mathscr{B}_{1} \subseteq \mathscr{A}_{1}^{a}$. As $\mathscr{B}=\mathscr{B}_{0}+\mathscr{B}_{1}$ and $\mathscr{A}_{1}$ generates $\mathscr{A}, \mathscr{A}_{1}^{a} \cap \mathscr{B}_{0}=0$ and $\mathscr{B}_{1}=\mathscr{A}_{1}^{a}$ and $\mathscr{B}_{0} \cap \mathscr{B}_{1}=0$. We now have to show that $\mathscr{B}_{1}$ generates $\mathscr{B}$. If this is not so there is a proper central projection $P \in \mathscr{B}_{0} \cap \mathscr{B}^{c}$ such that $P \mathscr{B}_{1}$ generates $P \mathscr{B}$ and $(1-P) \mathscr{B}_{1}=0$. Then $P \in \mathscr{A}_{0} \cap \mathscr{A}^{c}$ and $(1-P) \mathscr{B}_{0}$ $=(1-P) \mathscr{B}$, which on taking commutants gives $(1-P) \mathscr{A}=(1-P) \mathscr{A}_{0}$, which contradicts the assumption that $\mathscr{A}_{1}$ generates $\mathscr{A}$. This proves the existence of the opposed algebra. If $\mathscr{C}, \psi$ is the second opposed algebra then $\mathscr{C}=\mathscr{B}_{0}^{c}=\mathscr{A}, \mathscr{C}_{0}=\mathscr{B}^{c}$ $=\mathscr{A}_{0}$ and $\mathscr{C}_{1}=\mathscr{B}_{1}^{a}=\mathscr{A}_{1}^{a a} \supseteq \mathscr{A}_{1}$. As $\mathscr{C}=\mathscr{C}_{0}+\mathscr{C}_{1}$ is a direct sum decomposition, $\mathscr{C}_{1}=\mathscr{A}_{1}$ and the proof is complete.

Corollary 2.3. Let $\mathscr{S}$ be a set of operators $\mathscr{H}$ such that $A \in \mathscr{S}$ implies $A^{*} \in \mathscr{S}$ and $A \xi=0$ for all $A \in \mathscr{S}$ implies $\xi=0$. If there exists a symmetry $R$ of $\mathscr{H}$ such that $A R=-R A$ for all $A \in \mathscr{S}$ then $\mathscr{S}^{a a}$ is the closure in the weak operator topology of the odd polynomials in $\mathscr{S}$.

Proof. If $\mathscr{A}_{1}$ is the weak closure of the odd polynomials and $\mathscr{A}_{0}$ is the weak closure of the even polynomials without constant terms then the conditions imply that $\mathscr{A}_{0} \cap \mathscr{A}_{1}=0$ and that $\mathscr{A}_{0}$ contains the identity operator on $\mathscr{H}$, so $\mathscr{A}=\mathscr{A}_{0}+\mathscr{A}_{1}$ is a von Neumann algebra. Then $\mathscr{S}^{a} a=\mathscr{A}_{1}^{a a}=\mathscr{A}_{1}$. 
Now let $\mathscr{A}, \theta$ be any $W_{2}^{*}$-algebra and let $\mathscr{B}, \varphi$ be its opposed algebra. Then $\mathscr{A}_{0} \cap \mathscr{A}^{c}=\mathscr{B}_{0} \cap \mathscr{B}^{c}$ and we call this abelian algebra the centre of $\mathscr{A}$, or equivalently of $\mathscr{B}$. Any disjoint family of projections in $\mathscr{A}_{0} \cap \mathscr{A}^{c}$ with sum 1 effects a central decomposition of $\mathscr{A}$ into a sum of $W_{2}^{*}$-subalgebras on the corresponding subspaces of $\mathscr{H}$.

Clearly $\mathscr{A} \cap \mathscr{A}^{c}=\mathscr{A}_{0} \cap \mathscr{A}^{c}+\mathscr{A}_{1} \cap \mathscr{A}^{c}$ and if $\mathscr{U}$ is the von Neumann algebra generated by $\mathscr{A}_{1} \cap \mathscr{A}^{c}, \mathscr{U}$ is a weakly closed ideal in $\mathscr{A} \cap \mathscr{A}^{c}$ stable under $\theta$, so its identity element $P$ lies in $\mathscr{A}_{0} \cap \mathscr{A}^{c}$. Similarly $\mathscr{A}_{0} \cap \mathscr{A}_{0}^{c}=\mathscr{B} \cap \mathscr{B}^{c}=\mathscr{B}_{0} \cap \mathscr{B}^{c}$ $+\mathscr{B}_{1} \cap \mathscr{B}^{c}=\mathscr{A}^{c} \cap \mathscr{A}_{0}+\mathscr{A}_{1}^{a} \cap \mathscr{A}_{0}$ and if $\mathscr{V}$ is the von Neumann algebra generated by $\mathscr{A}_{1}^{a} \cap \mathscr{A}_{0}$ then $\mathscr{V}$ is a $\varphi$-stable weakly closed ideal in $\mathscr{B} \cap \mathscr{B}^{c}$ so its identity projection $Q$ lies in $\mathscr{B}_{0} \cap \mathscr{B}^{c}=\mathscr{A}_{0} \cap \mathscr{A}^{c}$. As $A B=B A=0$ for all $A \in \mathscr{A}_{1} \cap \mathscr{A}^{c}$ and $B \in \mathscr{A}_{1}^{a} \cap \mathscr{A}_{0}$ it follows that $P Q=Q P=0$. Now observe that

$$
\begin{aligned}
\mathscr{A} \cap \mathscr{A}_{0}^{c} & =\mathscr{B} \cap \mathscr{B}_{0}^{c}=\mathscr{B}_{0} \cap \mathscr{B}_{0}^{c}+\mathscr{B}_{1} \cap \mathscr{B}_{0}^{c}=\mathscr{A} \cap \mathscr{A}^{c}+\mathscr{A}_{1}^{a} \cap \mathscr{A} \\
& =\mathscr{A}_{0} \cap \mathscr{A}^{c}+\mathscr{A}_{1} \cap \mathscr{A}^{c}+\mathscr{A}_{0} \cap \mathscr{A}_{1}^{a}+\mathscr{A}_{1} \cap \mathscr{A}_{1}^{a} \\
& =\mathscr{A}_{0} \cap \mathscr{A}^{c}+\mathscr{A}_{1} \cap \mathscr{A}^{c}+\mathscr{A}_{0} \cap \mathscr{A}_{1}^{a} .
\end{aligned}
$$

We have now proved the following

PROPOSITION 2.4. Every $W_{2}^{*}$-algebra has a canonical central decomposition as a sum of the following three types:

Type A. $\mathscr{A}_{1} \cap \mathscr{A}_{1}^{c}=0, \mathscr{A}_{0} \cap \mathscr{A}_{1}^{a}$ generates $\mathscr{A}_{0} \cap \mathscr{A}_{0}^{c}, \mathscr{A} \cap \mathscr{A}^{c}=\mathscr{A}_{0} \cap \mathscr{A}^{c}$, $\mathscr{A} \cap \mathscr{A}_{0}^{c}=\mathscr{A}_{0} \cap \mathscr{A}_{0}^{c}$.

Type B. $\mathscr{A}_{0} \cap \mathscr{A}_{1}^{a}=0, \mathscr{A}_{1} \cap \mathscr{A}_{1}^{c}$ generates $\mathscr{A} \cap \mathscr{A}^{c}, \mathscr{A}_{0} \cap \mathscr{A}_{0}^{c}=\mathscr{A}_{0} \cap \mathscr{A}^{c}$, $\mathscr{A} \cap \mathscr{A}^{c}=\mathscr{A} \cap \mathscr{A}_{0}^{c}$.

Type C. $\mathscr{A}_{1} \cap \mathscr{A}_{1}^{c}=\mathscr{A}_{0} \cap \mathscr{A}_{1}^{a}=0, \mathscr{A}_{0} \cap \mathscr{A}_{1}^{c}=\mathscr{A}_{0} \cap \mathscr{A}_{0}^{c}=\mathscr{A} \cap \mathscr{A}^{c}=\mathscr{A} \cap \mathscr{A}_{0}^{c}$.

A $W_{2}^{*}$-algebra is of Type A, B, C if and only if its opposed algebra is of Type $\mathrm{B}, \mathrm{A}, \mathrm{C}$ respectively.

We call a $W_{2}^{*}$-algebra a factor if $\mathscr{A}_{0} \cap \mathscr{A}^{c}$ consists only of scalar multiples of the identity and see that every $W_{2}^{*}$-factor must be one of the three stated types. The structure of factors is clarified by the following

Proposition 2.5. The most general $W_{2}^{*}$-factors are described as follows:

Type A. $\mathscr{A}$ is a $W^{*}$-factor and $\theta$ is an inner automorphism.

Type B. $\mathscr{A}$ is the direct sum of $a W^{*}$-factor $\mathscr{B}$ with an isomorphic copy of $\mathscr{B}$ and $\theta$ interchanges terms of the two factors.

Type C. $\mathscr{A}$ is a $W^{*}$-factor and $\theta$ is an outer automorphism.

Proof. Suppose $\mathscr{A}, \theta$ is of Type B. Then $\mathscr{A} \cap \mathscr{A}^{c}$ is a commutative $\theta$-stable $C^{*}$-algebra of dimension greater than one such that the subspace of $\theta$-stable elements is of dimension one. It follows that $\mathscr{A} \cap \mathscr{A}^{c}$ has dimension two so $\mathscr{A}$ is the direct sum of two $W^{*}$-factors $\mathscr{B}$ and $\mathscr{C}$. As $\theta$ does not act trivially on $\mathscr{A} \cap \mathscr{A}^{c}$, $\theta$ exchanges $\mathscr{B}$ and $\mathscr{C}$ which must be isomorphic.

If $\mathscr{A}, \theta$ is of Type $\mathrm{A}$ or $\mathrm{C}$ then $\mathscr{A} \cap \mathscr{A}^{c}$ has dimension one, so $\mathscr{A}$ is a $W^{*}$-factor. If $\theta$ is inner there exists $R \in \mathscr{A}$ with $R=R^{*}, R^{2}=1$ and $\theta(X)=R X R$ for all $X \in \mathscr{A}$. 
Therefore $\mathscr{A}_{0} \cap \mathscr{A}_{0}^{c}$ has dimension two and $\mathscr{A}, \theta$ is of Type A. Conversely if $\mathscr{A}, \theta$ is of Type A the opposed algebra $\mathscr{B}, \varphi$ is of Type B so $\mathscr{A}_{0} \cap \mathscr{A}_{0}^{c}=\mathscr{B} \cap \mathscr{B}^{c}$ has dimension two. As $\mathscr{A}_{0} \cap \mathscr{A}_{0}^{c}=\mathscr{A}_{0} \cap \mathscr{A}^{c}+\mathscr{A}_{0} \cap \mathscr{A}_{1}^{a}$ and $\mathscr{A}_{0} \cap \mathscr{A}^{c}$ has dimension one there exists $R \in \mathscr{A}_{0} \cap \mathscr{A}_{1}^{a}$ such that $R=R^{*}$ and $R^{2}=1$. Then $R X=-X R$ for all $X \in \mathscr{A}_{1}$ so $\theta(X)=-X=R X R$ for all $X \in \mathscr{A}_{1}$ and $\theta(X)=R X R$ for all $X \in \mathscr{A}_{0}$. A special part of the following result was established in [16].

Proposition 2.6. Let $\mathscr{A}, \theta$ be a $W_{2}^{*}$-factor. Then $\mathscr{A}$ and $\mathscr{A}_{0}$ are $W^{*}$-algebras of the same general numerical type.

Proof. If $\mathscr{A}$ is semifinite with normal faithful semifinite trace $t$ then $s=t+t \theta$ is a normal faithful semifinite trace which is $\theta$-invariant. The restriction of $s$ to $\mathscr{A}_{0}$ is still semifinite. For if $0<A \in \mathscr{A}_{0}$ there exists $0<B \leqq A$ with $B \in \mathscr{A}$ and $s(B)<\infty$. If $C=\frac{1}{2}(B+\theta B)$ then $0<C \leqq A, C \in \mathscr{A}_{0}$ and $s(C)=s(B)<\infty$. Secondly one can show that $\mathscr{A}_{0}$ is discrete if and only if $\mathscr{A}$ is discrete. Finally if $\mathscr{A}$ is type III and $\mathscr{B}, \varphi$ is the opposed algebra then $\mathscr{B}_{0}=\mathscr{A}^{c}$ is type III by [6], so $\mathscr{B}$ must be type III, hence $\mathscr{A}_{0}$ must be type III.

We include the following result for completeness, although much more general theorems are known [11].

Proposition 2.7. Every involutory *automorphism of a Type $\mathrm{C} W_{2}^{*}$-factor is spatial.

Proof. We first observe that every *-automorphism of a discrete factor is inner so every Type $\mathrm{C}$ factor is continuous. By consideration of the opposed algebra it is sufficient to prove that if $\mathscr{A}, \theta$ is a Type $\mathrm{C} W_{2}^{*}$-factor there exists $R \in \mathscr{A}_{1}$ with $R=R^{*}$ and $R^{2}=1$.

Given any nonzero $A=A^{*}=-\theta A$ the spectral projections $P$ and $Q$ of $A$ corresponding to $\{\lambda: \lambda>0\}$ and $\{\lambda: \lambda<0\}$ satisfy $\theta P=Q$, so $P(\theta P)=(\theta P) P=0$ and $P \neq 0$. Let $P \geqq Q_{0}$ where $Q_{0}$ is a nonzero projection satisfying $\operatorname{tr}\left[Q_{0}\right]=(2 n)^{-1}$ if $\mathscr{A}$ is type $\mathrm{II}_{1}, \operatorname{tr}\left[Q_{0}\right]=1$ if $\mathscr{A}$ is type $\mathrm{II}_{\infty}$ and $Q_{0}=P$ if $\mathscr{A}$ is type III. Then $\mathscr{A}_{0}$ is a factor and $X_{0}=Q_{0}+\theta Q_{0}$ is a projection in $\mathscr{A}_{0}$ such that there exist $X_{1}, X_{2}, \ldots$ equivalent to $X_{0}$ under partial isometries in $U_{i}$ in $\mathscr{A}_{0}$, and $X_{0}+X_{1}+\cdots=1$. If $Q_{i}=U_{i}^{*} Q_{0} U_{i}$ then $Q_{i}\left(\theta Q_{i}\right)=\left(\theta Q_{i}\right) Q_{i}=0$ and $Q_{i}+\theta Q_{i}=X_{i}$. If $R=\left(Q_{0}-\theta Q_{0}\right)+\cdots+\left(Q_{i}-\theta Q_{i}\right)$ $+\cdots$ then $R=R^{*}=-\theta R$ and $R^{2}=1$, which completes the proof.

3. Tensor products of $W_{2}^{*}$-algebras. If $\mathscr{A}, \theta$ and $\mathscr{B}, \varphi$ are $W_{2}^{*}$-algebras acting on the Hilbert spaces $\mathscr{H}$ and $\mathscr{K}$ respectively then we define $\mathscr{A} \otimes \mathscr{B}$ as the $W^{*}$ algebra on $\mathscr{H} \otimes \mathscr{K}$ generated by all operators $A \otimes B$ where $A \in \mathscr{A}$ and $B \in \mathscr{B}$, [6]. Since the tensor product is in fact independent of the particular spaces $\mathscr{H}, \mathscr{K}$ used, by changing these so $\theta, \varphi$ become spatial, we see that there is a unique involutory automorphism $\theta \otimes \varphi$ of $\mathscr{A} \otimes \mathscr{B}$ such that $(\theta \otimes \varphi)(A \otimes B)=(\theta A) \otimes(\varphi B)$ for all $A \in \mathscr{A}$ and $B \in \mathscr{B}$. In this way $\mathscr{A} \otimes \mathscr{B}$ becomes a $W_{2}^{*}$-algebra and since

$$
(\mathscr{A} \otimes \mathscr{B}) \cap(\mathscr{A} \otimes \mathscr{B})^{c}=\left(\mathscr{A} \cap \mathscr{A}^{c}\right) \otimes\left(\mathscr{B} \cap \mathscr{B}^{c}\right)
$$


it follows that the tensor product of two $W_{2}^{*}$-factors is a $W_{2}^{*}$-factor unless both $\mathscr{A}$ and $\mathscr{B}$ are of Type $\mathrm{B}$. The only nontrivial question of type is solved in the following proposition which was obtained for a special case in [12].

Proposition 3.1. Let $\alpha$ and $\beta$ be ${ }^{*}$-automorphisms of the $W^{*}$-factors $\mathscr{A}$ and $\mathscr{B}$ respectively. Then $\alpha \otimes \beta$ is inner if and only if both $\alpha$ and $\beta$ are inner.

Proof. We show that if $\mathscr{A}$ acts on $\mathscr{H}, \mathscr{B}$ acts on $\mathscr{K}$ and $S$ is a unitary operator in $\mathscr{A} \otimes \mathscr{B}$ such that $(\alpha \otimes \beta) A=S^{*} A S$ for all $A \in \mathscr{A} \otimes \mathscr{B}$, then $\alpha$ is inner. There exist $\xi_{0}, \eta_{0} \in \mathscr{H} \quad$ and $\xi_{1}, \eta_{1} \in \mathscr{K}$ such that $\left\langle S\left(\xi_{0} \otimes \xi_{1}\right), \eta_{0} \otimes \eta_{1}\right\rangle \neq 0$. If $\lambda: \mathscr{L}(\mathscr{H} \otimes \mathscr{K}) \rightarrow \mathscr{L}(\mathscr{H})$ is defined by $\langle(\lambda A) \xi, \eta\rangle=\left\langle A\left(\xi \otimes \xi_{1}\right), \eta \otimes \eta_{1}\right\rangle$ then $\lambda$ is a bounded weakly continuous linear mapping and $T=\lambda S \neq 0$. Since $\lambda(A \otimes B)$ $=\left\langle B \xi_{1}, \eta_{1}\right\rangle A$ for all $A \in \mathscr{A}$ and $B \in \mathscr{B}, \lambda$ maps $\mathscr{A} \otimes \mathscr{B}$ onto $\mathscr{A}$. For all $A \in \mathscr{A}$ and $X \in \mathscr{L}(\mathscr{H} \otimes \mathscr{K}), \quad \lambda\{(A \otimes 1) X\}=A(\lambda X)$ and $\lambda\{X(A \otimes 1)\}=(\lambda X) A$, so for all $A \in \mathscr{A}, T(\alpha A)=A T$. Therefore for all $A \in \mathscr{A}$

$$
A T T^{*}=T(\alpha A) T^{*}=T\left(T \alpha A^{*}\right)^{*}=T\left(A^{*} T\right)^{*}=T T^{*} A
$$

and as $\mathscr{A}$ is a factor $T T^{*}=\alpha 1$ for some $\alpha \neq 0$. Similarly

$$
\begin{aligned}
A T^{*} T & =\left(T A^{*}\right)^{*} T=\left(\left(\alpha^{-1} A^{*}\right) T\right)^{*} T \\
& =T^{*}\left(\alpha^{-1} A\right) T=T^{*} T A
\end{aligned}
$$

so $T^{*} T=\beta 1$ for some $\beta \neq 0$. Then $\beta T=T T^{*} T=\alpha T$ so $\beta=\alpha$ and by normalising we can assume that $T T^{*}=T^{*} T=1$. Then for all $A \in \mathscr{A}, \alpha A=T^{*} A T$ so $\alpha$ is inner.

For $W_{2}^{*}$-algebras there is also a skew tensor product $\otimes_{2}$ whose definition is more complicated than that of $\otimes$, but which is more interesting. We leave the reader to verify that if $\mathscr{A}$ and $\mathscr{B}$ are finite dimensional then $\mathscr{A} \otimes_{2} \mathscr{B}$ coincides with the tensor product of $\mathscr{A}$ and $\mathscr{B}$ in the category of associative algebras graded over $\boldsymbol{Z}_{2}$, as described for example by Chevalley [2]. If $\mathscr{A}$ and $\mathscr{B}$ are $W_{2}^{*}$-algebras defined on $\mathscr{H}$ and $\mathscr{K}$ we define $\mathscr{D}_{i j}$ as the ultraweakly closed linear subspace of $\mathscr{A} \otimes \mathscr{B}$ generated by all $A \otimes B$ where $A \in \mathscr{A}_{i}$ and $B \in \mathscr{B}_{j}$ for $i=0,1$ and $j=0,1$. The products of elements of two $\mathscr{D}_{i j}$ lies in another of the $\mathscr{D}_{i j}$, for example $\mathscr{D}_{01} \cdot \mathscr{D}_{11} \subseteq \mathscr{D}_{10}$. Moreover the $\mathscr{D}_{i j}$ are disjoint, being alternatively defined by

$$
\mathscr{D}_{i j}=\left\{X \in \mathscr{A} \otimes \mathscr{B}:(\theta \otimes 1) X=(-)^{i} X \text { and }(1 \otimes \varphi) X=(-)^{j} X\right\}
$$

so that $\{\mathscr{A} \otimes \mathscr{B}\}_{0}=\mathscr{D}_{00}+\mathscr{D}_{11}$ and $\left\{\mathscr{A} \otimes \mathscr{B}_{1}=\mathscr{D}_{01}+\mathscr{D}_{10}\right.$. We identify $\mathscr{L}\left(\mathscr{H} \otimes \mathscr{K} \otimes C^{2}\right)$ with the set of $2 \times 2$ matrices with entries in $\mathscr{L}(\mathscr{H} \otimes \mathscr{K})$ and the obvious operations.

We define $\mathscr{C}=\mathscr{A} \otimes_{2} \mathscr{B} \subseteq \mathscr{L}\left(\mathscr{H} \otimes \mathscr{K} \otimes C^{2}\right)$ as the set

$$
\mathscr{C}=\left\{\left(\begin{array}{ll}
A+B & C+D \\
C-D & A-B
\end{array}\right): A \in \mathscr{D}_{00}, B \in \mathscr{D}_{10}, C \in \mathscr{D}_{01}, D \in \mathscr{D}_{11}\right\}
$$


and define

$$
\mathscr{C}_{0}=\left\{\left(\begin{array}{cc}
A & D \\
-D & A
\end{array}\right): A \in \mathscr{D}_{00}, D \in \mathscr{D}_{11}\right\}
$$

and

$$
\mathscr{C}_{1}=\left\{\left(\begin{array}{cc}
B & C \\
C & -B
\end{array}\right): B \in \mathscr{D}_{10}, C \in \mathscr{D}_{01}\right\}
$$

All of these are selfadjoint ultraweakly closed linear spaces of operators. Direct calculation yields $\mathscr{C}_{0} \cap \mathscr{C}_{1}=0, \mathscr{C}_{0}+\mathscr{C}_{1}=\mathscr{C}, \mathscr{C}_{0} \cdot \mathscr{C}_{0} \subseteq \mathscr{C}_{0}, \mathscr{C}_{0} \cdot \mathscr{C}_{1} \subseteq \mathscr{C}_{1}, \mathscr{C}_{1} \cdot \mathscr{C}_{0} \subseteq \mathscr{C}_{1}$, $\mathscr{C}_{1} \cdot \mathscr{C}_{1} \subseteq \mathscr{C}_{0}$. Therefore $\mathscr{C}$ is a $W^{*}$-algebra and there is a unique involutory ${ }_{\text {- }}$ automorphism $\psi$ of $\mathscr{C}$ such that $\psi\left(C_{0}+C_{1}\right)=C_{0}-C_{1}$ for all $C_{0} \in \mathscr{C}_{0}$ and $C_{1} \in \mathscr{C}_{1}$. There is a natural *-isomorphism $\pi$ of $\{\mathscr{A} \otimes \mathscr{B}\}_{0}$ onto $\mathscr{C}_{0}$ given by $\pi(A+D)$ $=\left(\begin{array}{cc}A & i D \\ -i D & { }_{A}\end{array}\right)$ for all $A \in \mathscr{D}_{00}$ and $D \in \mathscr{D}_{11}$. There is a natural *-isomorphism $\lambda$ of $\mathscr{A}$ into $\mathscr{C}$ given by

$$
\lambda\left(A_{0}+A_{1}\right)=\left(\begin{array}{cc}
A_{0} \otimes 1+A_{1} \otimes 1 & 0 \\
0 & A_{0} \otimes 1-A_{1} \otimes 1
\end{array}\right)
$$

for all $A_{0} \in \mathscr{A}_{0}$ and $A_{1} \in \mathscr{A}_{1}$, and $\lambda(\theta A)=\psi(\lambda A)$ for all $A \in \mathscr{A}$. There is a natural *-isomorphism $\mu$ of $\mathscr{B}$ into $\mathscr{C}$ given by

$$
\mu\left(B_{0}+B_{1}\right)=\left(\begin{array}{lllll}
1 \otimes B_{0} & 1 \otimes B_{1} \\
1 \otimes B_{1} & 1 \otimes B_{0}
\end{array}\right) \quad \text { for all } B_{0} \in \mathscr{B}_{0} \text { and } B_{1} \in \mathscr{B}_{1}
$$

and $\mu(\varphi B)=\psi(\mu B)$ for all $B \in \mathscr{B}$. If $A \in \mathscr{A}_{1}$ and $B \in \mathscr{B}_{1}$ then

$$
\lambda(A) \mu(B)=\left(\begin{array}{cc}
0 & A \otimes B \\
-A \otimes B & 0
\end{array}\right)=-(\mu B)(\lambda A)
$$

so that for all $A \in \mathscr{A}_{i}, B \in \mathscr{B}_{j}$, where $i=0,1$ and $j=0,1$,

$$
(\lambda A)(\mu B)=(-)^{i j}(\mu B)(\lambda A) .
$$

The $W^{*}$-algebra generated by $\mathscr{C}_{1}$ contains $\lambda\left(\mathscr{A}_{1}\right)$ and $\mu\left(\mathscr{B}_{1}\right)$ and hence $\lambda(\mathscr{A})$ and $\mu(\mathscr{B})$; therefore this $W^{*}$-algebra is $\mathscr{C}$. Noting that $\mathscr{A} \otimes \mathscr{B}$ is independent of the particular Hilbert spaces on which $\mathscr{A}$ and $\mathscr{B}$ act, the same is true of $\mathscr{A} \otimes_{2} \mathscr{B}$ and we have proved

Proposition 3.2. Given two $W_{2}^{*}$-algebras $\mathscr{A}$ and $\mathscr{B}$ there is a $W_{2}^{*}$-algebra $\mathscr{A} \otimes_{2} \mathscr{B}$ defined independently of the Hilbert spaces on which $\mathscr{A}$ and $\mathscr{B}$ act, with the following properties: $\mathscr{A}$ and $\mathscr{B}$ are embedded as $W_{2}^{*}$-subalgebras of $\mathscr{A} \otimes_{2} \mathscr{B}$ and generate $\mathscr{A} \otimes_{2} \mathscr{B}$. For all $A \in \mathscr{A}_{i}$ where $i=0,1$, and $B \in \mathscr{B}_{j}$, where $j=0,1$, we have $A B=(-)^{i j} B A .\left\{\mathscr{A} \otimes_{2} \mathscr{B}\right\}_{0}$ is isomorphic as a $W^{*}$-algebra with $\{\mathscr{A} \otimes \mathscr{B}\}_{0}$ and is the ultraweak closure of the linear subspace generated by all products $A B$ where 
$A \times B \in\left(\mathscr{A}_{0} \times \mathscr{B}_{0}\right) \cup\left(\mathscr{A}_{1} \times \mathscr{B}_{1}\right) .\left\{\mathscr{A} \otimes_{2} \mathscr{B}_{1}\right.$ is the ultraclosure of the linear subspace generated by all products $A B$ where $A \times B \in\left(\mathscr{A}_{0} \times \mathscr{B}_{1}\right) \cup\left(\mathscr{A}_{1} \times \mathscr{B}_{0}\right)$.

We can now indicate the relation of the skew tensor product to algebraic field theory [7]. For a mixed boson-fermion field we associate to every open bounded subset $U$ of space-time a $W^{*}$-algebra $\mathscr{F}(U)$, the field algebra, on a given Hilbert space. If $U \subseteq V$ then $\mathscr{F}(U) \subseteq \mathscr{F}(V)$ and we define $\mathscr{F}$ as the norm closure of the union of the $\mathscr{F}(U)$. The $C^{*}$-algebra $\mathscr{F}$ must have an involutory ${ }^{*}$-automorphism $\theta$ which leaves each of the algebras $\mathscr{F}(U)$ invariant, so we can write $\mathscr{F}(U)=\mathscr{F}_{0}(U)$ $+\mathscr{F}_{1}(U)$. If $U$ and $V$ are causally unrelated regions then for any $A \in \mathscr{F}_{i}(U)$, where $i=0,1$, and $B \in \mathscr{F}_{j}(V)$, where $j=0,1$, we suppose that $A B=(-)^{i j} B A$, as for the skew tensor product above. The field operators of fermion type associated with the region $U$ all lie in $\mathscr{F}_{1}(U)$ while the boson field operators and observables associated with $U$ lie in $\mathscr{F}_{0}(U)$. The $\mathscr{F}_{0}(U)$ form a system of local algebras in the sense of [10].

The above work also allows us to generalize Segal's theory of regular gauge space valued distributions [15] to arbitrary operator-valued distributions. If $\mathscr{H}$ is a real Hilbert space and $\mathscr{A}, \theta$ a $W_{2}^{*}$-algebra we define a (bounded reflection invariant) distribution to be a linear map from $\mathscr{H}$ to the selfadjoint elements of $\mathscr{A}$ with range contained in $\mathscr{A}_{1}$. If $T_{i}: \mathscr{H}_{i} \rightarrow \mathscr{A}^{i}, \theta^{i}$ are two distributions for $i=1,2$ then the skew product is the distribution $T=T_{1}+T_{2}: \mathscr{H}_{1}+\mathscr{H}_{2} \rightarrow \mathscr{A}^{1} \otimes_{2} \mathscr{A}^{2}$ and clearly satisfies $\left(T h_{1}\right)\left(T h_{2}\right)=-\left(T h_{2}\right)\left(T h_{1}\right)$ for all $h_{1} \in \mathscr{H}_{1}$ and $h_{2} \in \mathscr{H}_{2}$. Segal's finite trace on $\mathscr{A}, \theta$ can now be replaced by an arbitrary state, and we need not suppose that the von Neumann algebras involved are of finite type.

The following proposition will be used later but since its proof is rather routine we give it in outline only.

Proposition 3.3. If $\mathscr{A}, \mathscr{B}, \mathscr{C}$ are $W_{2}^{*}$-algebras there is a $W_{2}^{*}$-isomorphism of $\left(\mathscr{A} \otimes_{2} \mathscr{B}\right) \otimes_{2} \mathscr{C}$ onto $\mathscr{A} \otimes_{2}\left(\mathscr{B} \otimes_{2} \mathscr{C}\right)$ which identifies $\mathscr{A}, \mathscr{B}, \mathscr{C}$ under their natural embeddings as $W_{2}^{*}$-subalgebras of the two algebras.

Proof. Both the algebras consist of certain *-algebras of $4 \times 4$ matrices with values in $\mathscr{L}(\mathscr{H} \otimes \mathscr{K} \otimes \mathscr{M})$. For $i, j, k=0,1$ there are natural maps

$$
\lambda_{1}: \mathscr{A}_{i} \times \mathscr{B}_{j} \times \mathscr{C}_{k} \rightarrow\left(\mathscr{A} \otimes_{2} \mathscr{B}\right) \otimes_{2} \mathscr{C}
$$

and

$$
\lambda_{2}: \mathscr{A}_{i} \times \mathscr{B}_{j} \times \mathscr{C}_{k} \rightarrow \mathscr{A} \otimes_{2}\left(\mathscr{B} \otimes_{2} \mathscr{C}\right)
$$

It is sufficient to find a unitary map on $\boldsymbol{C}^{4}$, actually obtained by a permutation of the coefficients, such that if $U$ is the corresponding scalar-valued $4 \times 4$ matrix

$$
\lambda_{1}(A \times B \times C)=U^{*} \lambda_{2}(A \times B \times C) U
$$

for all $A \in \mathscr{A}_{i}, B \in \mathscr{B}_{j}, C \in \mathscr{C}_{k}$. 
As preparation for a more detailed study of the central structure of $\mathscr{C}=\mathscr{A} \otimes_{2} \mathscr{B}$ we note the following equations, obtained by direct computation:

$$
\begin{aligned}
& \mathscr{C}_{0} \cap \mathscr{C}^{c}=\left\{\left(\begin{array}{cc}
A & D \\
-D & A
\end{array}\right): A \in \mathscr{D}_{00} \cap(\mathscr{A} \otimes \mathscr{B})_{1}^{c}, D \in \mathscr{D}_{11} \cap(\mathscr{A} \otimes \mathscr{B})_{1}^{a}\right\} \\
& \mathscr{C}_{0} \cap \mathscr{C}_{1}^{a}=\left\{\left(\begin{array}{cc}
A & D \\
-D & A
\end{array}\right): A \in \mathscr{D}_{00} \cap(\mathscr{A} \otimes \mathscr{B})_{1}^{a}, D \in \mathscr{D}_{11} \cap(\mathscr{A} \otimes \mathscr{B})_{1}^{c}\right\} \\
& \mathscr{C}_{1} \cap \mathscr{C}_{1}^{c}=\left\{\left(\begin{array}{cc}
B & C \\
C & -B
\end{array}\right): B \in \mathscr{D}_{10} \cap \mathscr{D}_{10}^{c} \cap \mathscr{D}_{01}^{a}, C \in \mathscr{D}_{01} \cap \mathscr{D}_{01}^{a} \cap \mathscr{D}_{10}^{a}\right\}
\end{aligned}
$$

Proposition 3.4. If $\mathscr{A}, \theta$ and $\mathscr{B}, \varphi$ are Type $\mathrm{A} W_{2}^{*}$-factors then $\mathscr{A} \otimes_{2} \mathscr{B}$ is a Type A $W_{2}^{*}$-factor.

Proof. Let $\theta A=R A R$ and $B=S B S$ for all $A \in \mathscr{A}$ and $B \in \mathscr{B}$. Then $T=R \otimes S \in \mathscr{D}_{00}$ commutes with $\mathscr{D}_{00}, \mathscr{D}_{11}$ and anticommutes with $\mathscr{D}_{10}, \mathscr{D}_{01}$. Therefore

$$
\left(\begin{array}{ll}
T & \\
& T
\end{array}\right)\left(\begin{array}{ll}
A+B & C+D \\
C-D & A-B
\end{array}\right)\left(\begin{array}{ll}
T & \\
& T
\end{array}\right)=\left(\begin{array}{cc}
A-B & -C+D \\
-C-D & A+B
\end{array}\right)=\psi\left(\begin{array}{ll}
A+B & C+D \\
C-D & A-B
\end{array}\right)
$$

so $\psi$ is inner on $\mathscr{C} . \mathscr{A} \otimes \mathscr{B}$ is a factor of Type A so

$$
\mathscr{C}_{0} \cap \mathscr{C}_{0}^{c}=(\mathscr{A} \otimes \mathscr{B})_{0} \cap(\mathscr{A} \otimes \mathscr{B})_{0}^{c}
$$

has dimension two. Therefore $\mathscr{C}_{0} \cap \mathscr{C}^{c}$ has dimension one and $\mathscr{C}$ is a $W_{2}^{*}$-factor.

Proposition 3.5. If $\mathscr{A}, \theta$ is a Type $\mathrm{A} W_{2}^{*}$-factor and $\mathscr{B}, \varphi$ is a Type $\mathrm{B} W_{2}^{*}$-factor then $\mathscr{A} \otimes_{2} \mathscr{B}$ is a Type B $W_{2}^{*}$-factor.

Proof. Let $\theta A=R A R$ for all $A \in \mathscr{A}$ and let $\mathscr{B}$ be the direct product of the factor $\mathscr{B}_{1}$ with an isomorphic copy of itself. $\mathscr{A} \otimes \mathscr{B}_{1}$ can be represented by $2 \times 2$ matrices $\left(\begin{array}{ll}K & L \\ M & N\end{array}\right)$ with entries in $\mathscr{A} \otimes \mathscr{B}_{1}$ in such a way that $R \otimes 1$ is taken to $\left({ }^{1}-1\right)$; then every element of $\mathscr{C}$ is given by a pair of such matrices, and

$$
\begin{aligned}
& (\theta \otimes 1)\left\{\left(\begin{array}{ll}
K & L \\
M & N
\end{array}\right),\left(\begin{array}{ll}
K^{\prime} & L^{\prime} \\
M^{\prime} & N^{\prime}
\end{array}\right)\right\}=\left\{\left(\begin{array}{cc}
K & -L \\
-M & N
\end{array}\right),\left(\begin{array}{cc}
K^{\prime} & -L^{\prime} \\
-M^{\prime} & N^{\prime}
\end{array}\right)\right\} \\
& (1 \otimes \varphi)\left\{\left(\begin{array}{ll}
K & L \\
M & N
\end{array}\right),\left(\begin{array}{ll}
K^{\prime} & L^{\prime} \\
M^{\prime} & N^{\prime}
\end{array}\right)\right\}=\left\{\left(\begin{array}{cc}
K^{\prime} & L^{\prime} \\
M^{\prime} & N^{\prime}
\end{array}\right),\left(\begin{array}{ll}
K & L \\
M & N
\end{array}\right)\right\}
\end{aligned}
$$

Then $\left\{\left(\begin{array}{ll}{ }^{1} & 1\end{array}\right),\left(\begin{array}{ll}-1 & -1\end{array}\right)\right\} \in \mathscr{D}_{01}$ so $(\mathscr{A} \otimes \mathscr{B}) \cap \mathscr{D}_{01}^{a}=0$. Therefore $\mathscr{D}_{11} \cap(\mathscr{A} \otimes \mathscr{B})_{1}^{a}$, $\mathscr{D}_{00} \cap\left(\mathscr{A} \otimes \mathscr{B}_{1}^{a}\right.$ and $\mathscr{D}_{10} \cap \mathscr{D}_{10}^{c} \cap \mathscr{D}_{01}^{a}$ are all zero. Also $\mathscr{A} \otimes \mathscr{B}$ is a $W_{2}^{*}$-factor so

$$
(\mathscr{A} \otimes \mathscr{B})_{0} \cap(\mathscr{A} \otimes \mathscr{B})^{c}=\mathscr{D}_{00} \cap(\mathscr{A} \otimes \mathscr{B})_{1}^{c}+\mathscr{D}_{11} \cap(\mathscr{A} \otimes \mathscr{B})_{1}^{c}
$$

has dimension one. Therefore $\mathscr{C}_{0} \cap \mathscr{C}_{1}^{c}=C 1$ and $\mathscr{C}_{0} \cap \mathscr{C}_{1}^{a}=0$. Also one calculates that $\mathscr{D}_{01} \cap \mathscr{D}_{01}^{c} \cap \mathscr{D}_{10}^{a}$ has dimension one so $\mathscr{C}_{1} \cap \mathscr{C}_{1}^{c}$ has dimension one. Therefore $\mathscr{C}$ is a $W_{2}^{*}$-factor of Type B. 
Proposition 3.6. If $\mathscr{A}, \theta$ and $\mathscr{B}, \varphi$ are Type $\mathrm{B} W_{2}^{*}$-factors then $\mathscr{A} \otimes_{2} \mathscr{B}$ is a $W_{2}^{*}$-factor of Type A.

Proof. There exists a $W^{*}$-factor $\mathscr{E}$ such that $\mathscr{A} \otimes \mathscr{B}$ is the direct product of four isomorphic copies of $\mathscr{E}$ and

$$
\begin{aligned}
& (\theta \otimes 1)(K, L, M, N)=(L, K, N, M), \\
& (1 \otimes \varphi)(K, L, M, N)=(M, N, K, L) .
\end{aligned}
$$

Then $(1,-1,1,-1) \in \mathscr{D}_{10}$ so $(\mathscr{A} \otimes \mathscr{B}) \cap \mathscr{D}_{10}^{a}=0$. Also $(1,1,-1,-1) \in \mathscr{D}_{01}$ so $(\mathscr{A} \otimes \mathscr{B}) \cap \mathscr{D}_{01}^{a}=0 . \mathscr{D}_{11} \cap(\mathscr{A} \otimes \mathscr{B})_{1}^{c}=C(1,-1,-1,1)$ and $\mathscr{D}_{00} \cap(\mathscr{A} \otimes \mathscr{B})_{1}^{c}$ $=C(1,1,1,1)$. Therefore $\mathscr{C}_{1} \cap \mathscr{C}_{1}^{c}=0, \mathscr{C}_{0} \cap \mathscr{C}_{1}^{c}$ and $\mathscr{C}_{0} \cap \mathscr{C}_{1}^{a}$ have dimension one, and $\mathscr{C}$ is a Type $\mathrm{A} W_{2}^{*}$-factor.

Proposition 3.7. If $\mathscr{A}, \theta$ and $\mathscr{B}, \varphi$ are $W_{2}^{*}$-factors and either is Type $\mathrm{C}$ then $\mathscr{A} \otimes_{2} \mathscr{B}$ is a $W_{2}^{*}$-factor of Type C.

Proof. Suppose $\mathscr{B}$ is of Type C. Then by Proposition $3.1, \mathscr{A} \otimes \mathscr{B}$ is a $W_{2}^{*}$-factor of Type $\mathrm{C}$ so

$$
\mathscr{C}_{0} \cap \mathscr{C}_{0}^{c}=(\mathscr{A} \otimes \mathscr{B})_{0} \cap(\mathscr{A} \otimes \mathscr{B})_{0}^{c}
$$

has dimension one. By Proposition 2.4, $\mathscr{C}$ is a $W_{2}^{*}$-factor of Type B or C. If $\mathscr{A} \otimes_{2} \mathscr{B}$ is of Type $\mathrm{B}$ and $\mathscr{E}$ is a $W_{2}^{*}$-factor of Type B then $\left(\mathscr{D} \otimes_{2} \mathscr{A}\right) \otimes_{2} \mathscr{B}=\mathscr{D} \otimes_{2}\left(\mathscr{A} \otimes_{2} \mathscr{B}\right)$ is a $W_{2}^{*}$-factor of Type $\mathrm{A}$, which we have just shown cannot occur. Therefore $\mathscr{A} \otimes_{2} \mathscr{B}$ must be of Type $C$.

For comparison we now tabulate the types of $\mathscr{A}, \mathscr{B}, \mathscr{A} \otimes \mathscr{B}, \mathscr{A} \otimes_{2} \mathscr{B}$ together. If $\mathscr{A}$ and $\mathscr{B}$ are $W_{2}^{*}$-factors so are $\mathscr{A} \otimes \mathscr{B}$ and $\mathscr{A} \otimes_{2} \mathscr{B}$ in all but one case, indicated below:

$\begin{array}{cccc}\mathscr{A} & \mathscr{B} & \mathscr{A} \otimes \mathscr{B} & \mathscr{A} \otimes_{2} \mathscr{B} \\ A & A & A & A \\ A & B & B & B \\ B & B & B+B & A \\ A & C & C & C \\ B & C & B & C \\ C & C & C & C\end{array}$

As far as the numerical type is concerned the following considerations suffice for a complete solution. The numerical type of $\mathscr{A} \otimes \mathscr{B}$ can be calculated from that of $\mathscr{A}$ and $\mathscr{B}$ by [6], [14]. The numerical type of $\mathscr{A} \otimes \mathscr{B}$ is the same as that of $(\mathscr{A} \otimes \mathscr{B})_{0}$, and the numerical type of $\mathscr{A} \otimes_{2} \mathscr{B}$ is the same as that of $\left(\mathscr{A} \otimes_{2} \mathscr{B}\right)_{0}$, by Proposition 2.6. Moreover $(\mathscr{A} \otimes \mathscr{B})_{0}$ is isomorphic with $\left(\mathscr{A} \otimes_{2} \mathscr{B}\right)_{0}$ by Proposition 3.2.

We turn now to the study of the finite-dimensional $W_{2}^{*}$-algebras. For each integer $n$ there is exactly one Type B $W_{2}^{*}$-factor of dimension $2 n^{2}$, isomorphic as a $W^{*}$ - 
algebra with $\mathscr{M}(n, C) \oplus \mathscr{M}(n, C)$ with $\theta$ exchanging the terms of the two subalgebras. We call this factor $\mathscr{F}(n)$.

If $p, q$ are two positive integers and $n=p+q$, let $e_{i j}, 1 \leqq i, j \leqq n$, be a set of matrix units whose linear span is $\mathscr{M}(n, C)$. Let $R$ be the operator $R=\sum_{i=1}^{n} e_{i i}-\sum_{i=p+1}^{p} e_{i i}$, so $R=R^{*}$ and $R^{2}=1$, and let $\theta$ be defined by $\theta(X)=R X R$ for all $X \in \mathscr{M}(n, C)$. Then $\mathscr{M}(n, C), \theta$ describes the most general finite-dimensional Type $\mathrm{A} W_{2}^{*}$-factor and we denote it by $\mathscr{F}(p, q)$, or ty $\mathscr{F}\{|p-q|, p+q\}$. If $\tau$ is the positive trace on $\mathscr{M}(n, C)$ normalised by $\tau(1)=1$ then $\tau$ is $\theta$-invariant and $|\tau(R)|=|p-q|(p+q)^{-1}$ is called the discrepancy of $\mathscr{F}(p, q)$. Note that $\theta$ may be induced by $R$ or $-R$.

We define a set of matrix units for a finite-dimensional Type A $W_{2}^{*}$-factor to be a set of elements $e_{i j}, 1 \leqq i, j \leqq n$, whose linear span is the factor and such that $e_{i j} e_{k l}=\delta_{j k} e_{i l}, e_{i j}^{*}=e_{j i}, \sum_{i=1}^{n} e_{i i}=1$ and $\theta\left(e_{i j}\right)= \pm e_{i j}$. This last condition implies $\theta\left(e_{i i}\right)=e_{i i}$ for all $i$ and divides the index set $\{i: 1 \leqq i \leqq n\}$ into two equivalence classes such that $i, j$ are in opposite classes if and only if $\theta\left(e_{i j}\right)=-e_{i j}$.

Proposition 3.8.

$$
\begin{aligned}
\mathscr{F}(m) \otimes_{2} \mathscr{F}(n) & \simeq \mathscr{F}\{0,2 m n\}, \\
\mathscr{F}\{r, m\} \otimes_{2} \mathscr{F}(n) & \simeq \mathscr{F}(m n), \\
\mathscr{F}\{r, m\} \otimes_{2} \mathscr{F}\{s, n\} & \simeq \mathscr{F}\{r, m\} \otimes \mathscr{F}\{s, n\} \simeq \mathscr{F}\{r s, m n\} .
\end{aligned}
$$

Proof. All the above tensor products are finite-dimensional $W_{2}^{*}$-factors by our general analysis, and the exact type can be found from the following dimensional results, valid for arbitrary finite-dimensional $W_{2}^{*}$-algebras $\mathscr{A}$ and $\mathscr{B}$ :

$$
\begin{aligned}
& \operatorname{dim}(\mathscr{A} \otimes \mathscr{B})=\operatorname{dim}\left(\mathscr{A} \otimes_{2} \mathscr{B}\right)=\operatorname{dim} \mathscr{A} \operatorname{dim} \mathscr{B}, \\
& \operatorname{dim}(\mathscr{A} \otimes \mathscr{B})_{0}=\operatorname{dim}\left(\mathscr{A} \otimes_{2} \mathscr{B}\right)_{0}=\operatorname{dim} \mathscr{A}_{0} \operatorname{dim} \mathscr{B}_{0}+\operatorname{dim} \mathscr{A}_{1} \operatorname{dim} \mathscr{B}_{1}, \\
& \operatorname{dim}(\mathscr{A} \otimes \mathscr{B})_{1}=\operatorname{dim}\left(\mathscr{A} \otimes_{2} \mathscr{B}\right)_{1}=\operatorname{dim} \mathscr{A}_{0} \operatorname{dim} \mathscr{B}_{1}+\operatorname{dim} \mathscr{A}_{1} \operatorname{dim} \mathscr{B}_{0} .
\end{aligned}
$$

These equations come immediately from the definitions.

PROPOSITION 3.9. The algebra $\mathscr{F}\{r, n\}$ contains a $W_{2}^{*}$-subalgebra $\mathscr{B} \simeq \mathscr{F}\{s, m\}$ if and only if one of the following occurs:

(i) $r=s=0$ and $m \mid n$. $m$ and $n$ must both be even.

(ii) $r \neq 0, s \neq 0, s|r, m| n, r n^{-1} \leqq s m^{-1}$ and the integers $r s^{-1}$ and $m n^{-1}$ are both even or both odd.

Proof. If the conditions are satisfied we can construct such subalgebras using Proposition 3.8. Conversely suppose $\mathscr{F}\{r, n\}$ contains $\mathscr{B} \simeq \mathscr{F}\{s, m\}$. Then $\mathscr{A}=\mathscr{F}\{\boldsymbol{r}, n\} \cap \mathscr{B}^{c}$ is a $\theta$-invariant $W^{*}$-factor so either the action of $\theta$ on $\mathscr{A}$ is trivial or $\mathscr{A} \sim \mathscr{F}\{t, p\}$ for some integers $t \leqq p$ which are necessarily both even or both odd. $\mathscr{F}\{r, n\} \simeq \mathscr{A} \otimes \mathscr{B}$ in the $W_{2}^{*}$-algebra sense, from which the result follows.

4. Representations of $C_{2}^{*}$-algebras. In order to investigate further the structure of $W_{2}^{*}$-factors it is necessary to introduce the class of $C_{2}^{*}$-algebras and study their representations. 
We define a $C_{2}^{*}$-algebra as a $C^{*}$-algebra $\mathscr{A}$ together with an involutory *-automorphism $\theta$ such that $\mathscr{A}_{1}=\{A \in \mathscr{A}: \theta A=-A\}$ generates $\mathscr{A}$ in the norm topology. We caution the reader that because of the different topologies involved a $W_{2}^{*}$-algebra need not be a $C_{2}^{*}$-algebra. We always assume for simplicity that $\mathscr{A}$ has an identity element. We define a representation of $\mathscr{A}$ on a Hilbert space $\mathscr{H}$ to be a *-homomorphism $\lambda$ of $\mathscr{A}$ into the bounded operators on $\mathscr{H}$ such that there exists a symmetry $R$ on $\mathscr{H}$ (said to be associated with $\lambda$ ) such that $\lambda(\theta X)=R(\lambda X) R$ for all $X \in \mathscr{A}$. We also suppose $\lambda(1)=1$. If $\mathscr{B}_{i}$ is the weak closure of $\lambda\left(\mathscr{A}_{i}\right)$ for $i=0,1$ and $\mathscr{B}$ is the weak closure of $\lambda(\mathscr{A})$, then $\mathscr{B}, \mathscr{B}_{0}, \mathscr{B}_{1}$ define a $W_{2}^{*}$-algebra (called the enveloping $W_{2}^{*}$-algebra of the representation) whose involutive *automorphism is spatial, being induced by $R$. If $\lambda$ is a representation of $\mathscr{A}$ on $\mathscr{H}$ and $P$ is an orthogonal projection such that $P(\lambda X)=(\lambda X) P$ for all $X \in \mathscr{A}$, and $P R$ $=R P$ for some symmetry $R$ associated with $\lambda$, then $\lambda$ is said to be the direct sum of the subrepresentations obtained by restricting $\lambda$ to $P \mathscr{H}$ and $(1-P) \mathscr{H}$; this agrees with the obvious definition of the outer direct sum of two representations. A representation is said to be a factor representation if the enveloping $W_{2}^{*}$-algebra is a $W_{2}^{*}$-factor and to be irreducible if it cannot be written as a direct sum of proper subrepresentations in the above sense. If $\mathscr{A}$ is a $C_{2}^{*}$-algebra and $\lambda: \mathscr{A} \rightarrow \mathscr{L}(\mathscr{H})$, $\lambda^{\prime}: \mathscr{A} \rightarrow \mathscr{L}\left(\mathscr{H}^{\prime}\right)$ are two representations of $\mathscr{A}$, we say $\lambda$ and $\lambda^{\prime}$ are unitarily equivalent if there is a unitary operator $U: \mathscr{H} \rightarrow \mathscr{H}^{\prime}$ such that $\lambda(X)=U^{*}\left(\lambda^{\prime} X\right) U$ for all $X$ in $\mathscr{A}$. We say they are quasi-equivalent if there is a ${ }^{*}$-isomorphism $T$ of the weak closure $\mathscr{B}$ of $\lambda \mathscr{A}$ onto the weak closure $\mathscr{B}^{\prime}$ of $\lambda^{\prime} \mathscr{A}$ such that $T(\lambda X)=\lambda^{\prime} X$ for all $X$ in $\mathscr{A}$. Unitary equivalence implies quasi-equivalence and the ${ }^{*}$-isomorphism $T$ must necessarily be a $W_{2}^{*}$-isomorphism. Finally we say that $\lambda$ and $\lambda^{\prime}$ are algebraically equivalent if $\mathscr{B}$ and $\mathscr{B}^{\prime}$ are isomorphic as $W_{2}^{*}$-algebras.

We define a cyclic representation of $\mathscr{A}$ on $\mathscr{H}$ to consist of a ${ }^{*}$-homomorphism $\lambda: \mathscr{A} \rightarrow \mathscr{L}(\mathscr{H})$, a symmetry $R$ of $\mathscr{H}$ and a unit vector $\xi \in \mathscr{H}$ such that $R \xi=\xi$, $(\lambda \mathscr{A}) \xi$ is dense in $\mathscr{H}$, and $\lambda(\theta X)=R(\lambda X) R$ for all $X \in \mathscr{A}$. We define a state of $\mathscr{A}$ to be a positive linear functional $\varphi$ on $\mathscr{A}$ such that $\varphi(1)=1$ and $\varphi(\theta X)=\varphi(X)$ for all $X \in \mathscr{A}$. If $\lambda, R, \xi$ is a cyclic representation the functional $\varphi(X)=\langle(\lambda X) \xi, \xi\rangle$ is a state. Conversely every state of $\mathscr{A}$ defines a cyclic representation of $\mathscr{A}$ where $\mathscr{H}, \lambda$ and $\xi$ are given by the Gelfand-Segal construction and $R$ is defined by $R(\lambda X \xi)=\lambda X \xi$ for all $X \in \mathscr{A}_{0}$ and $R(\lambda X \xi)=-\lambda X \xi$ for all $X \in \mathscr{A}_{i}$. Finally if $\varphi$ is the state associated with a cyclic representation $\lambda, R, \xi$, the cyclic representation constructed from $\varphi$ is unitarily equivalent with $\lambda$. Every representation is a direct sum of cyclic representations so every irreducible representation is cyclic. We define a pure state of $\mathscr{A}$ to be a state $\varphi$ such that if $\psi$ is a $\theta$-invariant positive functional on $\mathscr{A}$ with $\psi \leqq \varphi$ then $\psi=\alpha \varphi$ for some real $\alpha, 0 \leqq \alpha \leqq 1$.

Proposition 4.1. Let $\lambda, R, \xi$ be a cyclic representation of a $C_{2}^{*}$-algebra $\mathscr{A}, \theta$ on $\mathscr{H}$. Then the following statements are equivalent:

(i) $\lambda \mathscr{A}, R$ generate the $W^{*}$-algebra $\mathscr{L}(\mathscr{H})$. 
(ii) $\varphi(X)=\langle\lambda X \xi, \xi\rangle$ is a pure state of $\mathscr{A}$.

(iii) $\lambda$ is an irreducible representation.

If $\lambda$ is an irreducible representation the enveloping $W_{2}^{*}$-algebra $\mathscr{B}, \theta$ of $\lambda \mathscr{A}$ is a $W_{2}^{*}$-factor of Type $\mathrm{A}$ or $\mathrm{B}$ and the propositions (Ai)-(Aiii) are equivalent, as are the propositions (Bi)-(Biii).

(Ai) The $W_{2}^{*}$-algebra opposed to $\mathscr{B}$ is isomorphic to $\mathscr{F}(1)$.

(Aii) $\lambda$ is an irreducible representation of the $C^{*}$-algebra $\mathscr{A}$ unitarily equivalent to the representation $\lambda \theta$.

(Aiii) $\lambda$ is an irreducible representation of $\mathscr{A}, \theta$ of Type $\mathrm{A}$.

(Bi) The $W_{2}^{*}$-algebra opposed to $\mathscr{B}$ is isomorphic to $\mathscr{F}(1,1)$.

(Bii) $\lambda=\mu+\mu \theta$ where $\mu$ is an irreducible representation of the $C^{*}$-algebra $\mathscr{A}$ which is not unitarily equivalent to $\mu \theta$.

(Biii) $\lambda$ is an irreducible representation of $\mathscr{A}, \theta$ of Type $\mathrm{B}$.

Proof. (iii) $\Rightarrow$ (i) is obvious. We prove (i) $\Leftrightarrow$ (ii), (i) $\Rightarrow$ (Ai) or (Bi), (Ai) $\Rightarrow$ (Aii) $\Rightarrow$ (Aiii) $\Rightarrow$ (iii) and $(\mathrm{Bi}) \Rightarrow$ (Bii) $\Rightarrow$ (Biii) $\Rightarrow$ (iii).

(i) $\Leftrightarrow$ (ii). There exists a one-one correspondence between the positive linear functionals $\psi$ on $\mathscr{A}$ with $\psi \leqq \varphi$ and the operators $A \in(\lambda \mathscr{A})^{\sim}$ with $0 \leqq A \leqq 1$ given by $\psi\left(Y^{*} X\right)=\langle A \lambda X \xi, \lambda Y \xi\rangle$ for all $X, Y \in \mathscr{A} . \psi$ is $\theta$-invariant if and only if $A R=R A$.

(i) $\Rightarrow$ (Ai) or (Bi). Let $\mathscr{C}, \psi$ be the $W_{2}^{*}$-algebra opposed to $\mathscr{B}, \varphi$ and observe that $R \in \mathscr{C}_{1}$. If $R=P-Q$ where $P, Q$ are orthogonal projections then $\psi P=Q$. If $C \in \mathscr{C}$ and $0 \leqq C \leqq P$ then $0 \leqq \psi C \leqq Q$ and $(C+\psi C) \in \mathscr{C}_{0}$ satisfies $(C+\psi C) R=R(C+\psi C)$. By hypothesis we conclude $C+\psi C=\alpha 1$ so $C=\alpha P$. It follows that for all $C \in \mathscr{C}$, $P C P=\alpha P$ and $Q C Q=\beta Q$. If $P C Q=0$ for all $C \in \mathscr{C}$, then $\mathscr{C}$ is generated by $P, Q$ and is isomorphic with $\mathscr{F}(1)$. On the other hand suppose there exists a nonzero $C \in \mathscr{C}$ with $C=P C=C Q$. If $D \in \mathscr{C}$ and $D=P D=D Q$ then $P D C^{*}=D C^{*}=D C^{*} P$ so $D C^{*}=\alpha P$ for some $\alpha$; similarly $C^{*} C=\beta Q$ for some $\beta \neq 0$. Then $D=D Q$ $=\beta^{-1} D C^{*} C=\beta^{-1} \alpha P C=\beta^{-1} \alpha C$. Therefore $\mathscr{C}$ is a four-dimensional $W^{*}$-factor with basis elements $P, Q, C, C^{*}$, and it follows that $\mathscr{C}, \psi \simeq \mathscr{F}(1,1)$.

(Ai) $\Rightarrow$ (Aii). $\lambda$ maps $\mathscr{A}$ onto a weakly dense subalgebra of $\mathscr{C}_{0}^{c}=(C 1)^{c}=\mathscr{L}(\mathscr{H})$ so $\lambda$ is an irreducible representation of the $C^{*}$-algebra $\mathscr{A} . \lambda \theta X=R(\lambda X) R$ for all $X \in \mathscr{A}$ so $\lambda$ is equivalent to $\lambda \theta$.

(Aii) $\Rightarrow$ (Aiii) $\Rightarrow$ (iii). For some unitary $U, \lambda \theta X=U(\lambda X) U^{*}$ for all $X \in \mathscr{A}$. Then $\lambda X=U^{2}(\lambda X) U^{* 2}$ and $\lambda$ is irreducible so $U^{2}=\alpha 1$. If $U= \pm \sqrt{ } \alpha R$ then $R=R^{*}$ and $R^{2}=1$ and $\lambda \theta X=R(\lambda X) R$ for all $X \in \mathscr{A}$. Therefore $\lambda$ is a representation in the $C_{2}^{*}$-algebra sense and it is irreducible of Type A.

(Bi) $\Rightarrow$ (Bii). In a suitable matrix presentation $\mathscr{C}$ is the set of all $2 \times 2$ scalar valued matrices, $R=\left(\begin{array}{ll}0 & 1 \\ 1 & 0\end{array}\right)$, and $\mathscr{A}=\mathscr{C}_{0}^{c}$ is the set of all matrices $\left(\begin{array}{ll}A & 0 \\ 0 & B\end{array}\right)$ where $A$ and $B$ are arbitrary. Therefore $\lambda X=\left(\begin{array}{cc}\mu X & 0 \\ 0 & v X\end{array}\right)$ for all $X \in \mathscr{A}$ where $\mu$ and $\nu$ are inequivalent irreducible representations. As $\lambda \theta X=R(\lambda X) R=\left(\begin{array}{cc}v X & 0 \\ 0 & \mu X\end{array}\right)$ we conclude $\nu=\mu \theta$.

(Bii) $\Rightarrow$ (Biii) $\Rightarrow$ (iii). $\lambda=\mu+\mu \theta$ is a representation in the $C_{2}^{*}$-algebra sense 
since in the above matrix presentation $R=\left(\begin{array}{ll}0 & 1 \\ 1 & 0\end{array}\right)$ is an associated symmetry. One verifies explicitly that $\mathscr{C}_{0}=\left\{\left(\begin{array}{cc}\alpha & 0 \\ 0 & \beta\end{array}\right): \alpha, \beta \in \boldsymbol{C}\right\}$ and $\mathscr{C}_{1}=\left\{\left(\begin{array}{ll}0 & \alpha \\ \beta & 0\end{array}\right): \alpha, \beta \in \boldsymbol{C}\right\}$ so $\mathscr{C} \simeq \mathscr{F}(1,1)$ and $\lambda$ is a representation of Type B. If $R$ is any symmetry associated with $\lambda$ then $R_{1} \in \mathscr{C}_{1}$ and

$$
R_{1}=\left(\begin{array}{cc}
0 & e^{i \theta} \\
e^{-i \theta} & 0
\end{array}\right) \text { for some } \theta
$$

Then $\left\{\lambda \mathscr{A}, R_{1}\right\}^{c}=\left\{X \in \mathscr{C}_{0}: R_{1} X=X R_{1}\right\}=C 1$ so $\lambda$ is irreducible.

Since the structure of Type A and Type B factors is quite clear, from now on we concentrate on the study of Type $C$ factors.

Proposition 4.2. Let $\mathscr{A}, \theta$ be a $C_{2}^{*}$-algebra and $\varphi$ a state of $\mathscr{A}$. Let $T \in \mathscr{A}_{1}$ satisfy $\varphi\left(T^{*} T\right)=1$ and let $\varphi_{1}$ be the restriction of $\varphi$ to $\mathscr{A}_{0}, \varphi_{2}$ the restriction of $X \rightarrow \varphi\left(T^{*} X T\right)$ to $\mathscr{A}_{0}$. Then $\varphi$ is a Type $\mathrm{C}$ factor state if and only if $\varphi$ is a factor state in the $C^{*}$-algebra sense, and $\varphi_{1}$ is a factor state of $\mathscr{A}_{0}$, and $\varphi_{1}$ is quasi-equivalent to $\varphi_{2}$ on $\mathscr{A}_{0}$.

Proof. Let $\lambda, R, \xi$ be the cyclic representation of $\mathscr{A}, \theta$ on $\mathscr{H}$ constructed from $\varphi$ and let $\mathscr{B}$ be the enveloping $W_{2}^{*}$-algebra of $\lambda \mathscr{A}$. If $\varphi$ is a factor state in the $C^{*}$ algebra sense then $\mathscr{B}$ is a $W_{2}^{*}$-factor of Type A or Type C. If $\mathscr{B}$ is of Type $\mathrm{C}$ then $\mathscr{B}_{0}=\left(\lambda \mathscr{A}_{0}\right)^{-}$is a factor and it is immediate that $\varphi_{1}$ and $\varphi_{2}$ are quasi-equivalent factor states of $\mathscr{A}_{0}$.

Suppose on the other hand that $\mathscr{B}$ is of Type A, and that $S=S^{*} \in \mathscr{B}, S^{2}=1$, implements the involutory *-automorphism of $\mathscr{B}$. In general $R \neq S$ but $S^{-1} R \in \mathscr{B}^{c}$ always. We can write $S=P-Q$ where $P, Q$ are disjoint central projections in $\mathscr{B}_{0}$. The two subrepresentations of $\mathscr{A}_{0}, \lambda_{1}$ on $P \mathscr{H}$ defined by $\lambda_{1} X=P(\lambda X) P$ and $\lambda_{2}$ on $Q \mathscr{H}$ defined by $\lambda_{2} X=Q(\lambda X) Q$, are disjoint, that is, not quasi-equivalent, factor representations. If $\xi_{1}=P \xi$ and $\xi_{2}=Q \xi$ are both nonzero vectors then for all $X \in \mathscr{A}_{0}$

$$
\varphi_{1}(X)=\left\langle\lambda X\left(\xi_{1}+\xi_{2}\right),\left(\xi_{1}+\xi_{2}\right)\right\rangle=\left\langle\lambda X \xi_{1}, \xi_{1}\right\rangle+\left\langle\lambda X \xi_{2}, \xi_{2}\right\rangle
$$

so $\varphi_{1}$ is a mixture of two disjoint factor states of $\mathscr{A}_{0}$ and is not a factor state. On the other hand if one of these vectors is zero, say $P \xi=\xi$, and if $\eta=\lambda T \xi$ then, because $T \in \mathscr{A}_{1}, Q_{\eta}=\eta$ and, because $\varphi\left(T^{*} T\right)=1,\|\eta\|=1$. The two states $\varphi_{1}$ and $\varphi_{2}$ induce the subrepresentations $\lambda_{1}$ and $\lambda_{2}$ respectively and so are disjoint.

Generalizing [9], we now define a U.H.F. $C_{2}^{*}$-algebra to be a $C_{2}^{*}$-algebra $\mathscr{A}, \theta$ which is the norm closure of an increasing sequence of finite-dimensional Type $\mathrm{A}$ $W_{2}^{*}$-subfactors $\mathscr{A}_{m}$. We suppose that $\mathscr{A}_{m} \simeq \mathscr{F}\left(p_{m}, q_{m}\right)$ and that the involutory *-automorphism of $\mathscr{A}_{m}$ is induced by $\pm R_{m} \in \mathscr{A}_{m}$. If we write $\mathscr{B}_{m}=\mathscr{A}_{m} \cap \mathscr{A}_{m-1}^{c}$ then $\mathscr{B}_{m}$ is a $W^{*}$-factor invariant under $\theta$. Either $\mathscr{B}_{m}$ is a $W_{2}^{*}$-factor with involutory *-automorphism induced by $S_{m} \in \mathscr{B}_{m}$ or the action of $\theta$ on $\mathscr{B}_{m}$ is trivial in which case we write $S_{m}=1 . \mathscr{A}_{m}$ can be identified with $\mathscr{A}_{m-1} \otimes \mathscr{B}_{m}$ and this is also a $W_{2}^{*}$ isomorphism with $R_{m}=R_{m-1} \otimes S_{m}$. If $\omega_{m}$ is a state on $\mathscr{B}_{m}$ (in the $W_{2}^{*}$-sense or an arbitrary state if $S_{m}=1$ ) there is a unique state $\omega$ on $\mathscr{A}$, which we call the product 
state, and which satisfies $\omega\left(B_{1} \cdots B_{n}\right)=\omega_{1}\left(B_{1}\right) \cdots \omega_{n}\left(B_{n}\right)$ for all $B_{i} \in \mathscr{B}_{i}$ and $i=1,2, \ldots$. We recall for reference the following important theorem of Powers [13].

Proposition 4.3. Let the $C^{*}$-algebra $\mathscr{C}$ be the norm closure of the increasing sequence of finite-dimensional $W^{*}$-subalgebras $\mathscr{C}_{n}$, all of these algebras having the same identity element, and let $\varphi_{1}, \varphi_{2}$ be two states on $\mathscr{C}$. Then $\varphi_{1}$ is a factor state if and only if it has the asymptotic product decomposition property and the factor states $\varphi_{1}$ and $\varphi_{2}$ are quasi-equivalent if and only if they are asymptotically equal.

We note that Powers' proof makes no essential use of his condition that the $\mathscr{C}_{n}$ be factors.

THEOREM 4.4. If $\omega$ is a product state on the U.H.F. $C_{2}^{*}$-algebra $\mathscr{A}$ then $\omega$ is a Type C factor state if and only if $\prod_{i=m}^{\infty}\left|\omega_{i}\left(S_{i}\right)\right|=0$ for all $m=1,2,3, \ldots$

Proof. Since $\omega$ has the asymptotic product decomposition property it is a factor state of Type A or C. If $\omega$ has the stated property we show that it satisfies the conditions of Proposition 4.2. Since $\mathscr{A}_{0}$ is the norm closure of the increasing sequence $\left(\mathscr{A}_{m}\right)_{0}$ of finite-dimensional subalgebras (not factors) we do this by using Proposition 4.3 on $\mathscr{A}_{0}$.

To prove that $\omega_{1}=\omega \mid \mathscr{A}_{0}$ has the asymptotic product decomposition property it is more than enough to prove that if $x \in \mathscr{A}_{n}$ and $\varepsilon>0$ there exists $m>n$ such that, for all $y \in \mathscr{A} \cap\left(\mathscr{A}_{m}\right)_{0}^{c},|\omega(x) \omega(y)-\omega(x y)|<\varepsilon\|y\|$. For any $m$ if $y \in \mathscr{A} \cap\left(\mathscr{A}_{m}\right)_{0}^{c}$, $y=\frac{1}{2}\left(1-R_{m}\right) y_{1}+\frac{1}{2}\left(1+R_{m}\right) y_{2}$ where $y_{1}, y_{2} \in \mathscr{A} \cap \mathscr{A}_{m}^{c}$ and $\left\|y_{1}\right\|,\left\|y_{2}\right\| \leqq\|y\|$. Also

$$
\omega(y)=\frac{1}{2} \omega\left(1-R_{m}\right) \omega\left(y_{1}\right)+\frac{1}{2} \omega\left(1+R_{m}\right) \omega\left(y_{2}\right)
$$

and

$$
\omega(x y)=\frac{1}{2} \omega\left\{x\left(1-R_{m}\right)\right\} \omega\left(y_{1}\right)+\frac{1}{2} \omega\left\{x\left(1+R_{m}\right)\right\} \omega\left(y_{2}\right)
$$

because $\omega$ is a product state. Now let $\left\{e_{i j}\right\}_{i, j=1}^{p_{n}+q_{n}}$ be a set of matrix units of $\mathscr{A}_{n}$ chosen so that

$$
R_{n}=\sum_{i=1}^{p_{n}} e_{i i}-\sum_{i=p_{n}+1}^{p_{n}+q_{n}} e_{i i} \text { and } \omega\left(e_{i j}\right)=\delta_{i j} \lambda_{i} .
$$

Then let $x=\sum_{i j} x_{i j} e_{i j}$ so $\omega(x)=\sum_{i=1}^{p_{n}+q_{n}} x_{i i} \lambda_{i}$ and observe that

$$
R_{m}=R_{n}\left(R_{n} R_{m}\right)=\sum_{i=1}^{p_{n}} e_{i i}\left(R_{n} R_{m}\right)-\sum_{i=p_{n}+1}^{p_{n}+q_{n}} e_{i i}\left(R_{n} R_{m}\right)
$$

and $R_{n} R_{m}=S_{n+1} \cdots S_{m}$ commutes with all the $e_{i i}$. Therefore

$$
\omega\left(x R_{m}\right)=\sum_{i=1}^{p_{n}} x_{i i} \lambda_{i} \omega\left(R_{n} R_{m}\right)-\sum_{i=p_{n}+1}^{p_{n}+a_{n}} x_{i i} \lambda_{i} \omega\left(R_{n} R_{m}\right)
$$


and $\left|\omega\left(R_{n} R_{m}\right)\right|=\prod_{i=n+1}^{m}\left|\omega_{i}\left(S_{i}\right)\right| \rightarrow 0$ as $m \rightarrow \infty$. Therefore given $\varepsilon>0$ by choosing $m$ large enough we obtain from (i) and (ii)

$$
\begin{aligned}
\left|\omega(y)-\frac{1}{2} \omega\left(y_{1}\right)-\frac{1}{2} \omega\left(y_{2}\right)\right| & <\frac{1}{2} \varepsilon\|x\|^{-1}\|y\|, \\
\left|\omega(x y)-\frac{1}{2} \omega(x) \omega\left(y_{1}\right)-\frac{1}{2} \omega\left(y_{2}\right)\right| & <\frac{1}{2} \varepsilon\|y\|
\end{aligned}
$$

which together imply

$$
|\omega(x y)-\omega(x) \omega(y)|<\varepsilon\|y\| \text {. }
$$

Now let $\left\{e_{i j}\right\}_{i, j=1}^{p_{1}+q_{1}}$ be a set of matrix units for $\mathscr{A}_{1}$ chosen so that $\omega\left(e_{i j}\right)=\delta_{i j} \mu_{i}$ where $\mu_{1} \neq 0$ and $\mu_{p_{1}+1} \neq 0$, and let $T=\alpha e_{1, p_{1}+1}$ where $\alpha$ is chosen so that $\omega\left(T^{*} T\right)=1$. We observe that $T \in \mathscr{A}_{1}$ and that the state $\omega^{\prime}(X)=\omega\left(T^{*} X T\right)$ is again a product state, the product of $\omega_{n}^{\prime}$ of $\mathscr{A}_{n}$ where $\omega_{n}^{\prime}=\omega_{n}$ for $n>1$. To prove that $\omega_{2}=\omega^{\prime} \mid \mathscr{A}_{0}$ is asymptotically equal to $\omega_{1}=\omega \mid \mathscr{A}_{0}$ it is more than enough to prove that for all $\varepsilon>0$ there exists $m$ so that $\left|\omega_{1}(y)-\omega_{2}(y)\right|<\varepsilon$ for all $y \in \mathscr{A} \cap\left(\mathscr{A}_{m}\right)_{0}^{c}$. Given $m$ and such a $y$ we can write $y=\frac{1}{2}\left(1+R_{m}\right) y_{1}+\frac{1}{2}\left(1-R_{m}\right) y_{2}$ where $y_{1}, y_{2} \in \mathscr{A} \cap \mathscr{A}_{m}^{c}$ and $\left\|y_{1}\right\|,\left\|y_{2}\right\| \leqq\|y\|$. Then

$$
\begin{aligned}
\mid \omega_{1}(y)- & \omega_{2}(y) \mid \\
& =\left|\frac{1}{2} \omega_{1}\left(R_{m}\right) \omega_{1}\left(y_{1}\right)-\frac{1}{2} \omega_{1}\left(R_{m}\right) \omega_{1}\left(y_{2}\right)-\frac{1}{2} \omega_{2}\left(R_{m}\right) \omega_{2}\left(y_{1}\right)+\frac{1}{2} \omega_{2}\left(R_{m}\right) \omega_{2}\left(y_{2}\right)\right| \\
& =\frac{1}{2}\left|\left\{\omega_{1}\left(R_{1}\right)-\omega_{1}^{\prime}\left(R_{1}\right)\right\} \omega\left(R_{1} R_{m}\right) \omega\left(y_{1}\right)+\left\{\omega_{1}^{\prime}\left(R_{1}\right)-\omega_{1}\left(R_{1}\right)\right\} \omega\left(R_{1} R_{m}\right) \omega\left(y_{2}\right)\right| \\
& \leqq 2\|y\|\left|\omega\left(R_{1} R_{m}\right)\right| \\
& =2\|y\| \sum_{i=2}^{m}\left|\omega_{i}\left(S_{i}\right)\right| \rightarrow 0 \text { as } m \rightarrow \infty
\end{aligned}
$$

We now prove that if all the stated equalities are not satisfied and if $p_{i}=q_{i}$ for all $i=1,2, \ldots$ then $\omega$ is of Type A. Suppose $\prod_{i=n+1}^{\infty}\left|\omega_{i}\left(S_{i}\right)\right|=\lambda>0$ and choose $x=R_{n} \in\left(\mathscr{A}_{n}\right)_{0}$. For any $m>n$ choose $y=\frac{1}{2}\left(1+R_{m}\right) \in \mathscr{A}_{0} \cap\left(\mathscr{A}_{m}\right)_{0}^{c}$. Let $\alpha=\omega\left\{\frac{1}{2}\left(1+R_{n}\right)\right\}$ so that $\omega(x)=2 \alpha-1$. Let

$$
\beta=\frac{1}{2} \omega\left(1+R_{n} R_{m}\right)
$$

so

$$
|2 \beta-1|=\left|\omega\left(S_{n+1} \cdots S_{m}\right)\right| \geqq \lambda .
$$

Then

$$
x y=\frac{1}{2} R_{n}\left(1+R_{m}\right)=\frac{1}{2}\left(1+R_{n}\right) \frac{1}{2}\left(1+R_{n} R_{m}\right)-\frac{1}{2}\left(1-R_{n}\right) \frac{1}{2}\left(1-R_{n} R_{m}\right)
$$

so $\omega(x y)=\alpha \beta-(1-\alpha)(1-\beta)$. Similarly

$$
y=\frac{1}{2}\left(1+R_{n}\right) \frac{1}{2}\left(1+R_{n} R_{m}\right)+\frac{1}{2}\left(1-R_{n}\right) \frac{1}{2}\left(1-R_{n} R_{m}\right)
$$

so $\omega(y)=\alpha \beta+(1-\alpha)(1-\beta)$. Therefore

$$
\begin{aligned}
|\omega(x y)-\omega(x) \omega(y)| & =|\alpha \beta-(1-\alpha)(1-\beta)-(2 \alpha-1)\{\alpha \beta+(1-\alpha)(1-\beta)\}| \\
& =|2 \alpha(1-\alpha)(2 \beta-1)| \geqq 2|\alpha(1-\alpha)| \lambda .
\end{aligned}
$$


Since $\alpha$ and $\lambda$ are independent of $m, \omega_{1}$ can only have the asymptotic product decomposition property if $\alpha=0$ or 1 . Let us suppose this happens. Then $\omega\left(R_{n}\right)$ $= \pm 1$ and because $\omega\left(R_{n}\right)=\omega\left(S_{1}\right) \cdots \omega\left(S_{n}\right)$ with $\left|\omega\left(S_{i}\right)\right| \leqq 1$ it follows that $\omega\left(R_{1}\right)$ $=\omega\left(S_{1}\right)= \pm 1$.

Using the fact that $p_{n}=q_{n}$, let $\left\{e_{i j}\right\}^{2 p_{n}}$ be a set of matrix units for $\mathscr{A}_{n}$ such that $R_{m}=\sum_{i=1}^{p_{n}}\left(e_{i i}-e_{p_{n}+i, p_{n}+i}\right)$ and $\omega\left(e_{i j}\right)=\delta_{i j} \lambda_{i}$. Then either $\lambda_{i}=0$ for all $i \leqq p_{n}$ or $\lambda_{i}=0$ for all $i \geqq p_{n}+1$.

Suppose for definiteness that the first of these possibilities occurs and define $T=\sum_{i=1}^{p_{n}} e_{i, p_{n}+i}$ so $\omega\left(T^{*} T\right)=1$ and $T \in\left(\mathscr{A}_{n}\right)_{1}$. We define $\omega_{2}$ as the restriction of $X \rightarrow \omega\left(T^{*} X T\right)$ to $\mathscr{A}_{0}$. For any $m>n$ we define $y=\frac{1}{2}\left(1+R_{m}\right) \in \mathscr{A}_{0} \cap\left(\mathscr{A}_{m}\right)_{0}^{c}$. Then

$$
y=\frac{1}{2}\left(1+R_{n}\right) \frac{1}{2}\left(1+R_{n} R_{m}\right)+\frac{1}{2}\left(1-R_{n}\right) \frac{1}{2}\left(1-R_{n} R_{m}\right)
$$

and

$$
T^{*} y T=\frac{1}{2}\left(1-R_{n}\right) \frac{1}{2}\left(1+R_{n} R_{m}\right)+\frac{1}{2}\left(1+R_{n}\right) \frac{1}{2}\left(1-R_{n} R_{m}\right)
$$

so

Therefore

$$
y-T^{*} y T=\frac{1}{2}\left(1+R_{n}\right) R_{n} R_{m}-\frac{1}{2}\left(1-R_{n}\right) R_{n} R_{m}=R_{m}
$$

$$
\begin{aligned}
\left|\omega_{1}(y)-\omega_{2}(y)\right| & =\left|\omega\left(y-T^{*} y T\right)\right| \\
& =\left|\omega\left(R_{m}\right)\right|=\left|\omega\left(R_{n}\right) \omega\left(R_{n} R_{m}\right)\right| \\
& =\left|\omega\left(R_{n} R_{m}\right)\right| \geqq \lambda>0
\end{aligned}
$$

so $\omega_{1}$ and $\omega_{2}$ cannot be asymptotically equal on $\mathscr{A}_{0}$.

We have now only to deal with the case where $p_{i} \neq q_{i}$ for some $i$. Let $\mathscr{B}_{i}^{\prime}=\mathscr{F}(1,1)$

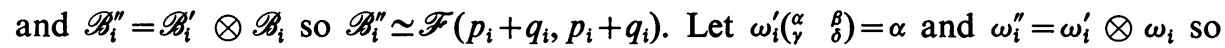
$\left|\omega_{i}^{\prime \prime}\left(S_{i}^{\prime \prime}\right)\right|=\left|\omega_{i}\left(S_{i}\right)\right|$. Let $\mathscr{A}^{\prime}$ be the infinite tensor product of $\left\{\mathscr{B}_{i}^{\prime}\right\}_{i=1}^{\infty}$ and $\mathscr{A}^{\prime \prime}$ the infinite tensor product of $\left\{\mathscr{B}_{i}^{\prime \prime}\right\}_{i=1}^{\infty}$ so $\mathscr{A}^{\prime \prime}=\mathscr{A}^{\prime} \otimes \mathscr{A}$ is a $C_{2}^{*}$-algebra and for the product states $\omega^{\prime \prime}=\omega^{\prime} \otimes \omega$. All these states are factor states and $\omega^{\prime}$ is a pure state and so is of Type A. By Proposition 3.1 applied to the enveloping $W_{2}^{*}$-algebras of the representations $\omega$ is of Type $C$ if and only if $\omega^{\prime \prime}$ is of Type C. But for the latter state we have computed the condition for this to happen, and the general result follows.

We finally include a result to show how badly behaved even a single involutory *-automorphism of a separable $C^{*}$-algebra can be. We shall need to use the theory of $\Sigma^{*}$-algebras and use the notation of [3], [4]. If $\mathscr{A}$ is a separable $C^{*}$ algebra its spectrum $\hat{A}$ has two Borel structures. The Mackey-Borel structure $\mathscr{M}$ is defined as the quotient Borel structure for the natural map $\nu: P(\mathscr{A}) \rightarrow \hat{\mathscr{A}}$ where $P(\mathscr{A})$ is the set of pure states of $\mathscr{A}$. The other Borel structure $\mathscr{B}$ is smaller than $\mathscr{M}$ and is defined in terms of the central elements of the $\sigma$-envelope $\mathscr{A}^{\sim}$ of $\mathscr{A}$. Effros [8] has shown that these need not be equal, although they are in case $\mathscr{A}$ is a separable G.C.R. algebra [3].

If $\theta$ is an involutory *-automorphism of $\mathscr{A}$, it induces an involutory automorphism $\theta^{\sim}$ of $\mathscr{A}^{\sim}$ and involutory Borel automorphisms, which we again call $\theta$, 
of $P(\mathscr{A}),(\hat{\mathscr{A}}, \mathscr{M})$ and $(\hat{\mathscr{A}}, \mathscr{B})$ in an evident fashion. By Proposition 4.1 the unitary equivalence classes of irreducible representations in the $C_{2}^{*}$-algebra sense correspond one-one to the $\theta$-orbits of $\hat{\mathscr{A}}$, each of which has either one or two points. Accordingly we define $\hat{\mathscr{A}_{A}}=\{\pi \in \hat{\mathscr{A}}: \theta \pi=\pi\}$ and $\hat{\mathscr{A}}_{B}=\{\pi \in \hat{\mathscr{A}}: \theta \pi \neq \pi\}$.

Proposition 4.5. The set $\hat{\mathscr{A}}_{A}$ is not necessarily a Borel set in $\hat{\mathscr{A}}$ for the Borel structure $\mathscr{B}$.

Proof. Let $\mathscr{A}, \theta$ be the U.H.F. $C_{2}^{*}$-algebra constructed as the norm closure of the union of an increasing family of finite-dimensional Type A $W_{2}^{*}$-subfactors $\mathscr{A}_{n}$, where $\mathscr{A}_{n} \cap \mathscr{A}_{n-1}^{c}=\mathscr{B}_{n} \simeq \mathscr{F}(1,1)$. Let $\alpha$ be a pure state of $\mathscr{F}(1,1)$ such that $\|\alpha-\alpha \theta\|=2$ and let $\alpha_{n}, \alpha_{n} \theta$ be the corresponding pure states of $\mathscr{B}_{n}$. Let $X$ $=\left\{\left(x_{n}\right)_{n=1}^{\infty}: x_{n}=0\right.$ or 1$\}$ and for $x \in X$ let $\varphi_{x}$ be the product state on $\mathscr{A}$ whose restriction to $\mathscr{B}_{n}$ is $\alpha_{n}$ or $\alpha_{n} \theta$ according as $x_{n}=0$ or 1 respectively. Let $\mu$ be the Haar measure of $X$ as a compact abelian group and for $x \in X$ let $(\theta x)_{n}=0$ or 1 according as $x_{n}=1$ or 0 respectively. It is known that $\varphi_{x}$ is a pure state of $\mathscr{A}$ and by Proposition $4.3, \varphi_{x}$ is not unitarily equivalent to $\varphi_{\theta x}$ for any $x \in X$. It is easy to show that for all $A \in \mathscr{A}, x \rightarrow \varphi_{x}(A)$ is continuous and

$$
\tau(A)=\int_{x} \varphi_{x}(A) \mu(d x)
$$

where $\tau$ is the unique normalised finite trace on $\mathscr{A}$. It follows that for all $A \in \mathscr{A}^{\sim}$, $x \rightarrow \varphi_{x}^{\tilde{x}}(A)$ is a bounded Borel function on $X$ and

$$
\tau^{\sim}(A)=\int \varphi_{x}^{\tilde{x}}(A) \mu(d x) .
$$

Similarly if $\beta$ and $\gamma$ are two states on $\mathscr{F}(1,1)$ such that $\beta=\beta \theta$ and $\gamma=\gamma \theta$ and $\|\beta-\gamma\|=2$ we let $\psi_{x}$ be the product state on $\mathscr{A}$ such that $\psi_{x} \mid \mathscr{B}_{n}=\beta$ or $\gamma$ according as $x_{n}=0$ or 1 respectively. $\psi_{x}=\psi_{\theta x}$ for all $x \in X$ and

$$
\tau^{\sim}(A)=\int_{x} \psi^{\sim}(A) \mu(d x)
$$

for all $A \in \mathscr{A}^{\sim}$. If $\hat{\mathscr{A}}_{A}$ were a Borel set in $\hat{\mathscr{A}}$ for $\mathscr{B}$ we could find a central projection $P \in \mathscr{A}^{\sim}$ such that $\varphi_{x}^{\tilde{x}}(P)=1$ for all $x \in X$ and $\psi_{\tilde{x}}(P)=0$ for all $x \in X$. But then $\tau^{\sim}(P)$ would equal zero and one, which is false.

5. Hyperfinite $W_{2}^{*}$-factors. The finer structure theory of U.H.F. $C_{2}^{*}$-algebras exhibits certain pathologies which do not occur in the theory of U.H.F. $C^{*}$ algebras [9] and it is necessary to clarify these before we can proceed. The basic difficulty is caused by the fact that $\mathscr{F}\{0, n\} \otimes \mathscr{F}\{r, m\} \simeq \mathscr{F}\{0, m n\}$ so that $\mathscr{F}\{0, n\}$ has many inequivalent embeddings in $\mathscr{F}\{0, m n\}$. Let the U.H.F. $C_{2}^{*}$-algebra $\mathscr{A}, \theta$ be the norm closure of the union of the increasing sequence of finite-dimensional Type A $W_{2}^{*}$-subfactors $\mathscr{A}_{m} \simeq \mathscr{F}\left\{\boldsymbol{r}_{m}, n_{m}\right\}$.

Proposition 5.1. If $\left\{e_{i j}\right\}_{i, j=1}^{p}$ is a set of matrix units in $\mathscr{A}$ and $\varepsilon>0$ then there exists an integer $q$ and matrix units $\left\{f_{i j}\right\}_{i, j=1}^{p}$ in $\mathscr{A}_{q}$ such that $\left\|e_{i j}-f_{i j}\right\|<\varepsilon$ and $\theta\left(f_{i j}\right)$ 
$= \pm f_{i j}$ according as $\theta\left(e_{i j}\right)= \pm e_{i j}$ respectively. Moreover there exists a unitary $u \in \mathscr{A}_{0}$ such that $u^{*} f_{i j} u=e_{i j}$ for $1 \leqq i, j \leqq p$ provided $\varepsilon>0$ is small enough.

Glimm's proof of the corresponding result for U.H.F. $C^{*}$-algebras [9] needs very minor modifications.

COROLlaRy 5.2. If $\mathscr{A}$ is a U.H.F. $C_{2}^{*}$-algebra and $\mathscr{B} \subseteq \mathscr{A}$ is a finite-dimensional Type A $W_{2}^{*}$-subfactor then either $\mathscr{A} \cap \mathscr{B}^{c}$ is a U.H.F. $C_{2}^{*}$-algebra or $\theta$ is trivial on $\mathscr{A} \cap \mathscr{B}^{c}$, in which case $\theta$ is inner on $\mathscr{A}$.

Proof. We can assume that $\mathscr{B} \subseteq \mathscr{A}_{n}$ for some $n$. Then $\mathscr{A} \cap \mathscr{B}^{c}$ is the norm closure of the union of $\mathscr{A}_{m} \cap \mathscr{B}^{c}$, which are $\theta$-stable finite-dimensional $W^{*}$-factors. Either the action of $\theta$ on all of these is trivial or they are all $W_{2}^{*}$-factors for large enough $m$. The result follows.

Let $\mathscr{A}, \theta$ be a U.H.F. $C_{2}^{*}$-algebra. We say $\mathscr{A}$ is degenerate if $\theta$ is inner. We say $\mathscr{A}$ is regular if it is nondegenerate and contains no $W_{2}^{*}$-subalgebra isomorphic to $\mathscr{F}\{0, n\}$ for any $n$. We say it is singular if it is neither degenerate nor regular. Finally we say it is completely singular if $\mathscr{A} \cap \mathscr{B}^{c}$ is singular for every finitedimensional Type A $W_{2}^{*}$-subfactor $\mathscr{B}$ of $\mathscr{A}$.

Proposition 5.3. The U.H.F. $C_{2}^{*}$-algebra $\mathscr{A}$ is regular if and only if $r_{m} \neq 0$ for all $m=1,2, \ldots$ and the monotonically decreasing sequence $\alpha_{m}=r_{m} n_{m}^{-1}$ is not eventually constant. If $\alpha=\lim \alpha_{m}$ and for all primes $p$ we define

$$
\begin{aligned}
& r(p)=\sup \left\{k: p^{k} \mid r_{m} \text { some } m\right\}, \\
& n(p)=\sup \left\{k: p^{k} \mid n_{m} \text { some } m\right\},
\end{aligned}
$$

then two regular U.H.F. $C_{2}^{*}$-algebras $\mathscr{A}$ and $\mathscr{A}^{\prime}$ are isomorphic if and only if $\alpha=\alpha^{\prime}$ and $r=r^{\prime}$ and $n=n^{\prime}$.

Proof. We follow Glimm's arguments [9] closely.

If $\mathscr{A}$ is regular then $r_{m} \neq 0$ for all $m$. Conversely if $\mathscr{A} \supseteq \mathscr{B} \simeq \mathscr{F}\{0, n\}$ then by Proposition 5.1, $\mathscr{A}_{m} \supseteq \mathscr{B}^{\prime} \simeq \mathscr{F}\{0, n\}$ for some $m$ and then $\mathscr{A}_{m} \simeq \mathscr{B}^{\prime} \otimes\left\{\mathscr{A}_{m} \cap\left(\mathscr{B}^{\prime}\right)^{c}\right\}$ so $r_{m}=0$ by Proposition 3.8. If $\alpha_{m+n}=\alpha_{n}$ for all $m \geqq 1$ then the action of $\theta$ on $\mathscr{A}_{m+n}$ $\cap \mathscr{A}_{n}^{c}$ is trivial so $R_{m+n}=R_{n}$ for all $m \geqq 1$ and $\theta$ is inner. Conversely if $\alpha_{m}$ is not eventually constant by passing to a subsequence we can assume $\alpha_{m+1}<\alpha_{m}$ for all $m$ so that the action of $\theta$ on each algebra $\mathscr{B}_{m}=\mathscr{A}_{m} \cap\left(\mathscr{A}_{m-1}\right)^{c}$ is nontrivial. If $\omega_{m}$ is a pure state on $\mathscr{B}_{m}$ such that $\left\|\omega_{m}-\omega_{m} \theta\right\|=2$ and $\omega$ is the product state, then $\omega$ is a pure state on the $C^{*}$-algebra $\mathscr{A}$ such that $\omega$ is not unitarily equivalent to $\omega \theta$. This shows that $\theta$ is not inner, and moreover proves that every nondegenerate U.H.F. $C_{2}^{*}$-algebra has an irreducible Type B representation.

If $\mathscr{A}$ is regular then the discrepancy $\alpha_{m}$ of $\mathscr{A}_{m}$ is characterised by

$$
1-\alpha_{m}=\sup \left\{\tau\left(A^{2}\right): A=A^{*}=-\theta A \text { and }\|A\| \leqq 1 \text { and } A \in \mathscr{A}_{m}\right\}
$$

so

$$
1-\alpha=\sup \left\{\tau\left(A^{2}\right): A=A^{*}=-\theta A \text { and }\|A\| \leqq 1 \text { and } A \in \mathscr{A}\right\} \text {. }
$$


Moreover by Proposition 5.1

$$
\{n(p), f(p)\}=\sup \left\{\{k, l\}: p^{k} \mid n \text { and } p^{l} \mid r \text { and } \mathscr{A} \supseteq \mathscr{B} \simeq \mathscr{F}\{r, n\}\right\},
$$

so $\alpha, n, r$ only depend on the isomorphism class of $\mathscr{A}$.

If $\mathscr{A}, \theta$ is regular with parameters $\alpha, n, r$ then, by Proposition $3.9, \mathscr{A}$, or equivalently $\bigcup_{m=1}^{\infty} \mathscr{A}_{m}$, contains a $W_{2}^{*}$-subalgebra isomorphic to $\mathscr{F}\{s, m\}$ if and only if $s m^{-1}>\alpha$ and $r(p)-\sup \left\{k: p^{k} \mid s\right\} \geqq 0$ and $n(p)-\sup \left\{k: p^{k} \mid m\right\} \geqq 0$, while for $p=2$ these last two expressions must also be either both zero or both finite nonzero or both infinite. The point is that this condition depends only on $\alpha, n, r$. Now suppose that $\mathscr{A}$ and $\mathscr{A}^{\prime}$ are two U.H.F. $C_{2}^{*}$-algebras with $\alpha=\alpha^{\prime}, n=n^{\prime}$ and $r=r^{\prime}$. Suppose $\lambda$ is an isomorphism of $\mathscr{A}_{i}$ into $\mathscr{A}_{j}^{\prime}$. Then $\mathscr{A} \cap \mathscr{A}_{i}^{c}$ is the closure of the union of $\mathscr{C}_{k}=\mathscr{A}_{i+k} \cap \mathscr{A}_{i}^{c}$ and is regular with parameters $\alpha \alpha_{i}^{-1}, r r_{i}^{-1}, n n_{i}^{-1}$. Similarly $\mathscr{A}^{\prime} \cap\left(\lambda \mathscr{A}_{i}\right)^{c}$ is the closure of the union of $\mathscr{C}_{k}^{\prime}=\mathscr{A}_{j+k} \cap\left(\lambda \mathscr{A}_{i}\right)^{c}$ and is regular with the same parameters, $\alpha \alpha_{i}^{-1}, r r_{i}^{-1}, n n_{i}^{-1}$. By our above observation there is an integer $k$ and an isomorphism $\mu$ of $\mathscr{C}_{1}^{\prime}$ into $\mathscr{C}_{k}$. Then $\mu \otimes \lambda^{-1}$ is an isomorphism of $\mathscr{A}_{j+1}^{\prime}$ into $\mathscr{A}_{i+k}$ which extends $\lambda^{-1}$. Repeating this procedure inductively provides an isomorphism of $\mathscr{A}$ with $\mathscr{A}^{\prime}$.

Proposition 5.4. The U.H.F. $C_{2}^{*}$-algebra $\mathscr{A}, \theta$ is completely singular if and only if there is a subsequence $m(i), i=1,2, \ldots$, such that $r_{m(i)}=0$ for all $i$ and

$$
\mathscr{A}_{m(i)} \cap \mathscr{A}_{m(i-1)}^{c} \simeq \mathscr{F}\left\{0, n_{m(i)} n_{m(i-1)}^{-1}\right\}
$$

for all $i$. If for all primes $p$ we define

$$
n(p)=\sup \left\{k: p^{k} \mid n_{m} \text { some } m\right\}
$$

then two completely singular U.H.F. $C_{2}^{*}$-algebras $\mathscr{A}$ and $\mathscr{A}^{\prime}$ are isomorphic if and only if $n=n^{\prime}$.

Proof. We call a sequence with the properties of $\mathscr{A}_{m(i)}$ a standard sequence. The proof that a completely singular algebra has the required properties follows from Proposition 5.1, and the converse follows as in the previous proposition. Similarly

$$
n(p)=\sup \left\{k: p^{k} \mid n \text { for some } \mathscr{F}\{0, n\} \simeq \mathscr{B} \subseteq \mathscr{A}\right\}
$$

and since for a standard sequence $n_{m} n_{m-1}^{-1}$ is even, $n(2)=\infty$. Therefore $\mathscr{A}$ contains a subalgebra isomorphic to $\mathscr{F}\{s, m\}$ if and only if $\sup \left\{k: p^{k} \mid m\right\} \leqq n(p)$ for all $p$, a criterion which depends only on $n$. The rest of the proof is as in the previous proposition.

Proposition 5.5. If $\mathscr{A}$ is a regular or completely singular U.H.F. $C_{2}^{*}$-algebra and if $\mathscr{B}_{1}$ and $\mathscr{B}_{2}$ are isomorphic finite-dimensional Type $\mathrm{A} W_{2}^{*}$-subfactors of $\mathscr{A}$ then $\mathscr{A} \cap \mathscr{B}_{1}^{c}$ and $\mathscr{A} \cap \mathscr{B}_{2}^{c}$ are isomorphic. If $\mathscr{A}$ is singular but not completely singular this may not be true.

Proof. If $\mathscr{A}$ is regular with parameters $\alpha, r, n$ and $\mathscr{B}_{1} \simeq \mathscr{F}\{s, m\}$ then $\mathscr{A} \cap \mathscr{B}_{i}^{c}$ are regular with parameters $\alpha^{\prime}, r^{\prime}, n^{\prime}$ given by $s \alpha^{\prime}=m \alpha$ and

$$
\begin{aligned}
& r(p)=r^{\prime}(p)+\sup \left\{k: p^{k} \mid s\right\}, \\
& n(p)=n^{\prime}(p)+\sup \left\{k: p^{k} \mid m\right\} .
\end{aligned}
$$


By Proposition 5.3 the two commutants are isomorphic. A similar argument works if $\mathscr{A}$ is completely singular.

If $\mathscr{A}$ is singular but not completely singular then for large enough $i$ the following holds. $\mathscr{A}_{i}$ has discrepancy zero and $\mathscr{A} \cap \mathscr{A}_{i}^{c}$ is regular with parameters $\alpha, r, n$. $\mathscr{A}_{i}$ contains two subalgebras $\mathscr{B}_{1}, \mathscr{B}_{2}$ both isomorphic to $\mathscr{F}\{0, m\}$ with $\mathscr{A}_{i} \cap \mathscr{B}_{1}^{c}$ $\simeq \mathscr{F}\left\{r, n_{i} m^{-1}\right\}$ and $\mathscr{A}_{i} \cap \mathscr{B}_{2}^{c} \simeq \mathscr{F}\left\{s, n_{i} m^{-1}\right\}$ with $r \neq s, r \neq 0$ and $s \neq 0$. Then $\mathscr{A} \cap \mathscr{B}_{1}^{c}$ and $\mathscr{A} \cap \mathscr{B}_{2}^{c}$ are regular with parameters $\alpha_{1}, r_{1}, n_{1}$ and $\alpha_{2}, r_{2}, n_{2}$ satisfying $\alpha_{1}$ $=r n_{i} m^{-1} \alpha$ and $\alpha_{2}=s n_{i} m^{-1} \alpha$. If $\alpha \neq 0$ then $\alpha_{1} \neq \alpha_{2}$ and the two commutators are not isomorphic.

Proposition 5.6. Let $\omega_{1}$ and $\omega_{2}$ be two Type C factor states of a U.H.F. $C_{2}^{*-}$ algebra $\mathscr{A}$ which is regular or completely singular. Then $\omega_{1}$ and $\omega_{2}$ are algebraically equivalent if and only if there is an automorphism $\nu$ of $\mathscr{A}$ (as a $C_{2}^{*}$-algebra) such that $\omega_{1}$ and $\omega_{2} \nu$ are quasi-equivalent.

Proof. This important theorem is due to Powers [13]. If $\mathscr{B}, \theta$ is the enveloping $W_{2}^{*}$-algebra of the representation we must be careful to construct all the unitary operators of [13, Lemmas 3.1-3.6] to lie in $\mathscr{B}_{0}$. In [13, Lemma 3.3] we must assume that $\mathscr{B}$ is a Type $\mathrm{C} W_{2}^{*}$-factor in order to use the conclusion that $\mathscr{B}_{0}$ is a factor, and also make use of Proposition 2.6.

Now let us suppose that $\mathscr{A}, \theta$ is a U.H.F. $C_{2}^{*}$-algebra and $\mathscr{A}_{m} \cap \mathscr{A}_{m-1}^{c}$ $=\mathscr{B}_{m} \simeq \mathscr{F}\{\boldsymbol{r}, \boldsymbol{n}\}$ for all $m$, so $\mathscr{A}$ is regular if $r \neq 0$ and completely singular if $r=0$. Suppose $\omega$ is the product of the states $\omega_{m}$ on $\mathscr{B}_{m}$, all of which correspond to the same state $\omega_{0}$ on $\mathscr{F}\{r, n\}$. If $R$ induces the involutive automorphism of $\mathscr{F}\{r, n\}$ then, by Theorem $4.4, \omega$ is a Type $C$ factor state of $\mathscr{A}$ if and only if $\left|\omega_{0}(R)\right| \neq 1$. Let $\sigma$ be constructed in the same way from another state $\sigma_{0}$ on $\mathscr{F}\{r, n\}$.

Proposition 5.7. If $\omega$ and $\sigma$ are algebraically equivalent states then for any $\varepsilon>0$ there exists an integer $k$ such that the following holds. Suppose $\mathscr{B}$ is a $W_{2}^{*}$-factor isomorphic to $\mathscr{F}\left\{\boldsymbol{r}^{k}, n^{k}\right\}$ and $\mathscr{N}_{i}, i=1, \ldots, k$, is a factorisation of $\mathscr{B}$ into $W_{2}^{*-}$ subfactors with isomorphisms $\lambda_{i}: \mathscr{N}_{i} \rightarrow \mathscr{F}\{r, k\}$. Let $\omega_{i}=\omega_{0} \lambda_{i}$ on $\mathscr{N}_{i}$ and let $\omega^{\prime}$ be the product state on $\mathscr{B}$. There exists a factorisation $\mathscr{M}_{1}, \mathscr{M}_{2}$ of $\mathscr{B}$, states $\rho_{1}, \rho_{2}$ on $\mathscr{M}_{1}, \mathscr{M}_{2}$ respectively, and an isomorphism $\mu: \mathscr{M}_{1} \rightarrow \mathscr{F}\{r, n\}$ such that $\rho_{1}=\sigma_{0} \mu$ and $\left\|\rho_{1} \otimes \rho_{2}-\omega^{\prime}\right\|<\varepsilon$.

This is again obtained by minor modifications of Powers' proof [13].

From now on we shall restrict our analysis to the completely singular U.H.F. algebra $\mathscr{A}, \theta$ whose parameter $n$ is given by $n(2)=\infty$ and $n(p)=0$ for $p \neq 2$. If $\varphi$ is any state on $\mathscr{F}\{0,2 n\}$ then matrix units $e_{i j}$ can be chosen for $\mathscr{F}\{0,2 n\}$ so that $\varphi\left(e_{i j}\right)=\delta_{i j} \lambda_{i}$. If the involutory automorphism of $\mathscr{F}\{0,2 n\}$ is induced by $R=\sum_{i=1}^{n}\left(e_{i i}-e_{n+i, n+i}\right)$, the set of eigenvalues with multiplicities is divided into two classes according as $i \geqq n+1$ or $i \leqq n$. The multiplicity of each eigenvalue in each class is independent of the particular set of matrix units chosen, although the two classes are interchanged if we replace $R$ by $-R$. 
For any integer $k$ by taking a suitable subsequence of the generating algebras we can assume that each of the algebras $\mathscr{B}_{i}=\mathscr{A}_{i} \cap \mathscr{A}_{i-1}^{c}$ is isomorphic to $\mathscr{F}\left\{0,2^{k}\right\}$. If $0<\lambda<\frac{1}{2}$ let $\rho_{0}$ be a state on $\mathscr{F}\left\{0,2^{k}\right\}$ whose eigenvalues are $\lambda^{i}(1-\lambda)^{k-i}$ with multiplicities $(k !)\{(i !)(k-i) !\}^{-1}$, the eigenvalues being divided into two classes of equal size by a set of matrix units of $\mathscr{F}\left\{0,2^{k}\right\}$ chosen to diagonalise $\rho_{0}$. Let $\rho$ be the product state on $\mathscr{A}, \theta$ whose restriction to each $\mathscr{B}_{i}$ is carried onto $\rho_{0}$ by the isomorphism of $\mathscr{F}\left\{0,2^{k}\right\}$ with $\mathscr{B}_{i}$. Under these circumstances we call $\rho$ a product state of $\mathscr{A}, \theta$ associated with $\lambda, 0<\lambda<\frac{1}{2}$.

THEOREM 5.9. The product states of $\mathscr{A}, \theta$ associated with each $\lambda, 0<\lambda<\frac{1}{2}$, fall into exactly two algebraic equivalence classes. Each of the hyperfinite type III factors of Powers has at least two involutory outer automorphisms which are not conjugate under the group of all automorphisms of the factor.

Proof. We first take $k=2$, so $\mathscr{B}_{i}=\mathscr{A}_{i} \cap \mathscr{A}_{i-1}^{c}$ is isomorphic to $\mathscr{F}\{0,4\}$. Let $\left\{e_{i j}\right\}_{i, j=1}^{4}$ be matrix units for $\mathscr{F}\{0,4\}$ so that the involutory *-automorphism of $\mathscr{F}\{0,4\}$ is induced by $R=e_{11}+e_{22}-e_{33}-e_{44}$. Let $\omega_{0}$ be the state

$$
\omega_{0}\left(\sum_{i, j=1}^{4} x_{i j} e_{i j}\right)=\lambda(1-\lambda) x_{11}+\lambda(1-\lambda) x_{22}+\lambda^{2} x_{33}+(1-\lambda)^{2} x_{44},
$$

and let $\sigma_{0}$ be the state

$$
\sigma_{0}\left(\sum_{i, j=1}^{4} x_{i j} e_{i j}\right)=\lambda^{2} x_{11}+\lambda(1-\lambda) x_{22}+\lambda(1-\lambda) x_{33}+(1-\lambda)^{2} x_{44} .
$$

We prove that the conclusion of Proposition 5.7 is invalid for $\varepsilon=\lambda^{2}$, so that the product states $\omega$ and $\sigma$ on $\mathscr{A}, \theta$ are not algebraically equivalent in the $C_{2}^{*}$-algebra sense. Since they are clearly algebraically equivalent in the $C^{*}$-algebra sense this proves the second statement of the theorem.

Using the notation of Proposition 5.7 it is immediate that the eigenvalues of $\omega^{\prime}$ on $\mathscr{B}$ are $\lambda^{i}(1-\lambda)^{2 k-i}$ with multiplicities $(2 k) !\{(i !)(2 k-i) !\}^{-1}$ and that these are divided into two classes according as $i$ is even or odd. Let us call this set of eigenvalues $T$ and let $\mu_{1}, \ldots, \mu_{l}$ denote the eigenvalues of $\rho_{2}$ on $\mathscr{M}_{2}$, with multiplicities included, so the eigenvalues of $\rho_{1} \otimes \rho_{2}$ are $\lambda^{2} \mu_{i}, \lambda(1-\lambda) \mu_{i}, \lambda(1-\lambda) \mu_{i},(1-\lambda)^{2} \mu_{i}$ where $1 \leqq i \leqq l$, multiplicities being included. Comparing the eigenvalue lists of $\omega^{\prime}$ and $\rho_{1} \otimes \rho_{2}$ separately for the two classes we obtain as in [13]

$$
\left\|\rho_{1} \otimes \rho_{2}-\omega^{\prime}\right\| \geqq \sum_{i=1}^{l} s_{i}
$$

where

$$
\begin{aligned}
s_{i} & =\left|\lambda^{2} \mu_{i}-t_{i 1}\right|+\left|\lambda(1-\lambda) \mu_{i}-t_{i 2}\right|+\left|\lambda(1-\lambda) \mu_{i}-t_{i 3}\right|+\left|(1-\lambda)^{2} \mu_{i}-t_{i 4}\right| \\
& \geqq\left|t_{i 2}-t_{i 3}\right|
\end{aligned}
$$

and where $t_{i j}, 1 \leqq j \leqq 4,1 \leqq i \leqq l$, is a suitable parametrisation of the elements of $T$. 
For each $i$ the two eigenvalues $\lambda(1-\lambda) \mu_{i}$ are of opposite class, so $t_{i 2}$ and $t_{i 3}$ are of opposite class. Therefore

$$
\left|t_{i 2}-t_{i 3}\right| \geqq \max \left\{t_{i 2}, t_{i 3}\right\} \lambda(1-\lambda)^{-1} .
$$

We also have by hypothesis

$$
\begin{aligned}
\lambda^{2}>\left\|\rho_{1} \otimes \rho_{2}-\omega^{\prime}\right\| & \geqq \sum_{i=1}^{l} s_{i} \geqq \sum_{i=1}^{l}\left|2 \lambda(1-\lambda) \mu_{i}-t_{i 2}-t_{i 3}\right| \\
& \geqq \sum_{i=1}^{l}\left\{2 \lambda(1-\lambda) \mu_{i}-\left(t_{i 2}+t_{i 3}\right)\right\} \\
& =2 \lambda(1-\lambda)-\sum_{i=1}^{l}\left(t_{i 2}+t_{i 3}\right)
\end{aligned}
$$

so

$$
\begin{aligned}
\sum_{i=1}^{l} \max \left\{t_{i 2}, t_{i 3}\right\} & \geqq \sum_{i=1}^{l}\left(t_{i 2}+t_{i 3}\right) \\
& \geqq 2 \lambda(1-\lambda)-\lambda^{2}>\lambda(1-\lambda) .
\end{aligned}
$$

Therefore

$$
\left\|\rho_{1} \otimes \rho_{2}-\omega^{\prime}\right\|>\sum_{i=1}^{l} \max \left\{t_{i 2}, t_{i 3}\right\} \lambda(1-\lambda)^{-1}>\lambda^{2}
$$

which contradicts the above hypothesis.

To prove the first part of the theorem let $\rho$ be a product state constructed from $\rho_{0}$ on $\mathscr{F}\{0,2 k\}$. If the eigenvalues $\lambda^{i}(1-\lambda)^{k-i}$ are divided into two classes according as $i$ is even or odd then it follows quickly from Proposition 5.6 that $\rho$ is algebraically equivalent to $\omega$. We show that if this is not so then $\rho$ is algebraically equivalent to $\sigma$.

We suppose there exist integers $i, j$ either both even or both odd and eigenvalues $\lambda^{i}(1-\lambda)^{k-i}$ and $\lambda^{j}(1-\lambda)^{k-j}$ of opposite classes. If $i+j=2 x$ then

$$
\left\{\lambda^{x}(1-\lambda)^{k-x}\right\}^{2}=\lambda^{i}(1-\lambda)^{k-i} \cdot \lambda^{j}(1-\lambda)^{k-j}
$$

so $\lambda^{2 x}(1-\lambda)^{2 k-2 x}$ occurs in both classes of the eigenvalue list of $\rho \mid \mathscr{A}_{i} \cap \mathscr{A}_{i-2}^{c}$ for every $i$. It is therefore sufficient to consider the case where $k$ is even and the two classes of eigenvalue lists of $\rho$, say $\mu_{1}, \ldots, \mu_{y}$ and $\mu_{y+1}, \ldots, \mu_{2 y}$, have some common entry, say $\mu_{1}=\mu_{y+1}$. Here each $\mu_{i}$ is of the form $\lambda^{j}(1-\lambda)^{k-j}$ and $2 y=2^{k}$.

Let $\varepsilon>0$ and let $n$ be any integer. The eigenvalues of $\rho \mid \mathscr{A}_{n}$ are $\mu_{1}^{r_{1}} \cdots \mu_{2 y}^{r_{2 y}}$ where $r_{1}+\cdots+r_{2 y}=n$. We can write $\left\{\left(r_{1}, r_{y+1}\right): 0 \leqq r_{1}+r_{y+1} \leqq n\right\}$ as a disjoint union of sets $S_{1}, \ldots, S_{t}, S$ such that each set $S_{i}$ consists of two points

$$
\left\{\left(r_{1}, r_{y+1}+1\right),\left(r_{1}+1, r_{y+1}\right)\right\} \text { and } S \subseteq\left\{\left(r_{1}, r_{y+1}\right): r_{1}=0\right\} .
$$

For each $r_{2}, \ldots, r_{y}, r_{y+2}, \ldots, r_{2 y}$ and each set $S_{i}$ the two eigenvalues

and

$$
\mu_{1}^{r_{1}} \cdots \mu_{y}^{r_{y}} \mu_{y+1}^{r_{y}+1+1} \mu_{y+2}^{r_{y}+2} \cdots \mu_{2 y}^{r_{2 y}}
$$

$$
\mu_{1}^{r_{1}+1} \mu_{2}^{r_{2}} \cdots \mu_{2 y}^{r_{2 y}}
$$


are equal numerically but lie in opposite classes. Moreover the sum of the remaining eigenvalues is not greater than the constant coefficient of

$$
\left(\mu_{1} x+\mu_{2}+\cdots+\mu_{2 y}\right)^{n}=\left(1-\mu_{1}\right)^{n}+x f(x) .
$$

Therefore for sufficiently large $n$ we can divide the eigenvalues of $\rho \mid \mathscr{A}_{n}$ into three sets $T, U, V$ such that the eigenvalues in $T, U$ are the same with the same multiplicities but lie in opposite classes, and the sum of the eigenvalues in $V$ is less than $\varepsilon / 4$. If the multiplicity of the eigenvalue $\lambda_{i}=\lambda^{i}(1-\lambda)^{n k-i}$ in $T$ (or in $U$ ) is $n_{i}$ then we have shown that

$$
2 n_{i} \leqq(n k) !\{(i !)(n k-i) !\}^{-1}
$$

and

$$
\sum_{i=0}^{n k}\left[(n k) !\{(i !)(n k-i) !\}^{-1}-2 n_{i}\right] \lambda^{i}(1-\lambda)^{n k-i}<\varepsilon / 4 .
$$

Similar arguments apply to the state $\sigma$ on $\mathscr{A}, \theta$ and for large enough $n$ we can find sets $T^{\prime}, U^{\prime}, V^{\prime}$ such that if the multiplicity of $\lambda_{i}$ in $T^{\prime}$ (or in $U^{\prime}$ ) is $m_{i}$ then

and

$$
2 m_{i} \leqq(n k) !\{(i !)(n k-i) !\}^{-1}
$$

$$
\sum_{i=0}^{n k}\left[(n k) !\{(i !)(n k-i) !\}^{-1}-2 m_{i}\right] \lambda^{i}(1-\lambda)^{n k-i}<\varepsilon / 4 .
$$

If $\rho_{i}=\min \left(m_{i}, n_{i}\right)$ then

$$
\sum_{i=0}^{n k}\left[(n k) !\{(i !)(n k-i) !\}^{-1}-2 \rho_{i}\right] \lambda^{i}(1-\lambda)^{n k-i}<\varepsilon / 2
$$

and $T, U, T^{\prime}, U^{\prime}$ each contain subsets $T_{1}, U_{1}, T_{1}^{\prime}, U_{1}^{\prime}$ such that the eigenvalue $\lambda_{i}$ occurs in each subset with multiplicity $\rho_{i}$. The eigenvalues of $T_{1}$ and $U_{1}$ (as of $T_{1}^{\prime}$ and $U_{1}^{\prime}$ ) lie in opposite classes so by changing the notation if necessary we can assume that the eigenvalues in $T_{1}$ and $T_{1}^{\prime}$ (or in $U_{1}$ and $U_{1}^{\prime}$ ) lie in the same class. It is now straightforward to construct a unitary operator $U \in\left\{\mathscr{A}_{n}\right\}_{0}$ such that

$$
\left\|\sigma(\cdot)-\rho\left(U^{*} \cdot U\right) \mid \mathscr{A}_{n}\right\|<\varepsilon .
$$

Exactly the same argument can be applied to the restriction of $\rho$ and $\sigma$ to $\mathscr{A} \cap \mathscr{A}_{n}^{c}$. Inductively it follows that there exists a sequence $m_{n}$ and a sequence of unitary operators $U_{n} \in\left\{\mathscr{A}_{m_{n}}\right\}_{0} \cap\left\{\mathscr{A}_{m_{n-1}}\right\}^{c}$ such that

$$
\left\|\sigma(\cdot)-\rho\left(U_{n}^{*} \cdot U_{n}\right) \mid \mathscr{A}_{m_{n}} \cap\left\{\mathscr{A}_{m_{n-1}}\right\}^{c}\right\|<1 / 2^{n} .
$$

If $\alpha$ is the unique *-automorphism of $\mathscr{A}$ such that $\alpha(X)=U_{n}^{*} X U_{n}$ for all $X \in \mathscr{A}_{m_{n}} \cap\left\{\mathscr{A}_{m_{n-1}}\right\}^{c}$ and $\rho^{\prime}(X)=\rho(\alpha X)$ then $\rho^{\prime}$ is algebraically equivalent to $\rho$ and

$$
\left\|\left(\sigma-\rho^{\prime}\right) \mid \mathscr{A}_{m_{n}} \cap\left\{\mathscr{A}_{m_{n-1}}\right\}^{c}\right\|<1 / 2^{n} .
$$

The proof is completed by proving that $\sigma$ and $\rho^{\prime}$ are asymptotically equal, and so quasi-equivalent. 
Let $\rho_{n}$ be the state on $\mathscr{A}$ which has a product decomposition given by

$$
\begin{array}{lll}
\rho_{n}^{\prime}=\sigma & \text { on } \mathscr{A}_{m_{i}} \cap\left\{\mathscr{A}_{m_{i-1}}\right\}^{c} & \text { for } i<n, \\
\rho_{n}^{\prime}=\rho^{\prime} & \text { on } \mathscr{A}_{m_{i}} \cap\left\{\mathscr{A}_{m_{i-1}}\right\}^{c} & \text { for } i \geqq n .
\end{array}
$$

It is easy to check that $\left\|\rho_{n}^{\prime}-\rho_{n+1}^{\prime}\right\|<2^{1-n}$ and also that $\sigma-\rho^{\prime}=\sum_{n=1}^{\infty}\left(\rho_{n}-\rho_{n-1}\right)$, the limit certainly existing in the weak* topology of $\mathscr{A}^{*}$. Therefore

$$
\left\|\left(\sigma-\rho^{\prime}\right) \mid \mathscr{A} \cap\left\{\mathscr{A}_{m_{i}}\right\}^{c}\right\| \leqq \sum_{n=1}^{\infty} 2^{1-i}=2^{2-n}
$$

so $\sigma$ and $\rho^{\prime}$ are indeed asymptotically equal.

We leave as an open question whether each of the hyperfinite type III factors has an infinite number of nonconjugate involutory automorphisms. We also leave the interested reader to work out for himself the much easier problem for the hyperfinite $\mathrm{II}_{1}$ factor. The classification of the involutory inner automorphisms is of course trivial.

6. Group representations. In this section we show how our study of $C_{2}^{*}$ algebras and $W_{2}^{*}$-algebras is related to the theory of group representations and give some illustrative examples. To do this we have to introduce the idea of an epigroup and, since this seems to be new even at the algebraic level, we do this more generally than will be needed in the application.

An epigroup $(G, \varphi, z)$ over a ring $R$ is defined as a group $G$ together with a homomorphism $\varphi$ on $G$ onto $(R+)$; we define $G_{0}=\operatorname{ker} \varphi$ and suppose $G_{1}=G \backslash G_{0}$ generates the group $G$; we also suppose $r \rightarrow z^{r}$ is an isomorphism of $(R+)$ into the centre of $G$ with range contained in $G_{0}$. If $R$ has an identity element 1 we write $z^{1}=z$. If $(G, \varphi, z)$ is an epigroup we define a homomorphism $\alpha$ from $(R+)$ to Aut $(G)$ by $\alpha_{r}(g)=z^{\varphi(g)} \cdot g$ and write $\alpha_{1}=\alpha$ if $R$ has an identity element.

If $(G, \varphi, z)$ and $(H, \psi, \omega)$ are two epigroups over $R$ we define their skew product. The definition

$$
(g, h)\left(g_{1}, h_{1}\right)=\left(g g_{1}, \omega^{(\psi h) \cdot\left(\varphi g_{1}\right)} h h_{1}\right)
$$

makes the set $G \times H$ into a group. The subset $\left\{\left(z^{r}, \omega^{s}\right): r, s \in R\right\}$ is a central subgroup and we define the skew product $G \times{ }_{2} H$ as the quotient group of $G \times H$ under the homomorphism $\pi: G \times H \rightarrow G \times{ }_{2} H$ whose kernel $k$ is $\left\{\left(z^{r}, \omega^{-r}\right): r \in R\right\}$. We define $X: G \times{ }_{2} H \rightarrow(R+)$ by $X\{\pi(g, h)\}=\varphi(g)+\psi(h)$ and define $x:(R+) \rightarrow G \times{ }_{2} H$ by

$$
x^{r}=\pi\left(z^{r}, e\right)=\pi\left(e, \omega^{r}\right) .
$$

These definitions make $\left(G \times{ }_{2} H, X, x\right)$ into an epigroup over $R$ and the maps $g \rightarrow \pi(g, e)$ and $h \rightarrow \pi(e, h)$ are one-one embeddings of $G, H$ as subepigroups of $G \times{ }_{2} H$ in an obvious sense. $G \times{ }_{2} H$ is generated by $G, H$ subject to the relations $z^{r}=\omega^{r}$ for all $r \in R$ and $h g=\omega^{(\psi h) \cdot(\varphi g)} g h$ for all $g \in G, h \in H$.

We define a 2-epigroup $(G, \varphi, z)$ to be an epigroup over $R=Z_{2}$ such that $G$ is a 
separable locally compact group and both $G_{0}$ and $G_{1}$ are closed. We define a representation $U$ of a 2 -epigroup $(G, \varphi, z)$ on a Hilbert space $\mathscr{H}$ to be a unitary representation such that $U_{z}=-1$ and such that there exists a symmetry $R$ on $\mathscr{H}$ such that $U_{g} R=-R U_{g}$ for all $g \in G_{1}$, or equivalently $U_{\alpha g}=R U_{g} R$ for all $g \in G$. We then define

$$
\mathscr{A}_{i}=\varlimsup_{\operatorname{lin}}\left\{U_{g_{i}}: g_{i} \in G_{i}\right\}
$$

for $i=0,1$ where $\overline{\text { in }}$ is the weak operator closed linear span. Then for all $A_{i} \in \mathscr{A}_{i}$, $A_{i} R=(-)^{i} R A_{i}$ so $\mathscr{A}_{1} \cap \mathscr{A}_{0}=0$ and $\mathscr{A}=\mathscr{A}_{0}+\mathscr{A}_{1}$ is a $W_{2}^{*}$-algebra which we call the enveloping algebra of the representation. The type of the representation is identified with the type of $\mathscr{A}$. It is easy to define the skew tensor product of two representations of two 2-epigroups in such a way as to correspond naturally to our definition of the skew tensor product of two $W_{2}^{*}$-algebras. We now give some examples of 2-epigroups.

EXAMPLE 1. Let Pin $(n)$ be the double covering of $O(n)$ constructed in [1] and let $\psi: \operatorname{Pin}(n) \rightarrow Z_{2}$ be the homomorphism whose kernel is Spin $(n)$. Let $\omega$ be the central element of Pin $(n)$ of order two which maps to the identity under the covering map of Pin $(n)$ onto $O(n)$. Let $G$ be the semidirect product of Pin $(n)$ with $\boldsymbol{R}^{n}$ under the obvious action of $O(n)$ on $R^{n}$. Let $z$ be the element of $G$ corresponding to $\omega ; z$ is central of order two since $\omega$ acts trivially on $\boldsymbol{R}^{n}$. Let $\varphi$ be the homomorphism of $G$ onto $Z_{2}$ whose kernel is the semidirect product of Spin $(n)$ with $R^{n}$. Then $G$ is a 2-epigroup and its irreducible representations can be calculated from Proposition 4.1 and the theory of induced representations.

EXAMPLE 2. Let $G_{0}=\operatorname{SL}(2 n, R)$ and let $\beta: G_{0} \rightarrow G_{0}$ be the Cartan involution $\beta(A)=\left(A^{T}\right)^{-1}$. Then we define $G$ as the semidirect product of $G_{0}$ by $\beta$ and let $\varphi: G \rightarrow Z_{2}$ be the homomorphism with kernel $G_{0}$. If we define $z=-1$ then $(G, \varphi, z)$ is a 2-epigroup. It is possible to associate such a Lie 2-epigroup canonically with several of the symmetric spaces in this way.

EXAMPLE 3. If $(G, \varphi, z)$ is an abelian 2-epigroup then $(\hat{G}, \hat{\varphi}, \hat{z})$ is an abelian 2-epigroup if we define $\hat{\varphi}=z$ and $\hat{z}=\varphi$.

\section{REFERENCES}

1. M. F. Atiyah, R. Bott and A. Shapiro, Clifford modules, Topology 3 (1964), suppl. 1, 3-38. MR 29 \#5250.

2. C. Chevalley, The construction and study of certain important algebras, Math. Soc. Japan, Tokyo, 1955. MR 17, 342.

3. E. B. Davies, On the Borel structure of $C^{*}$-algebras, Comm. Math. Phys. 8 (1968), 147-163. MR 37 \#6764.

4. - The structure of $\Sigma^{*}$-algebras, Quart. J. Math. Oxford Ser. (2) 20 (1969), 351-366.

5. J. Dixmier, Les $C^{*}$-algèbres et leurs représentations, 2ième éd., Cahiers Scientifiques, fasc. 29, Gauthier-Villars, Paris, 1969. MR 39 \#7442.

6. ——, Les algèbres d'opérateurs dans l'espace Hilbertien, 2ième éd., Cahiers Scientifiques, fasc. 25, Gauthier-Villars, Paris, 1969. 
7. S. Doplicher, R. Haag and J. F. Roberts, Fields, observables and gauge transformations. I, Comm. Math. Phys. 13 (1969), 1-23.

8. E. G. Effros, The canonical measures for a separable C*-algebra, Amer. J. Math. 92 (1970), 56-60.

9. J. G. Glimm, On a certain class of operator algebras, Trans. Amer. Math. Soc. 95 (1960), 318-340. MR 22 \#2915.

10. R. Haag and D. Kastler, An algebraic approach to quantum field theory, J. Mathematical Phys. 5 (1964), 848-861. MR 29 \#3144.

11. R. V. Kadison, Unitary invariants for representations of operator algebras, Ann. of Math. (2) 66 (1957), 304-379. MR 19, 665.

12. M. Nakamura and Z. Takeda, On outer automorphisms of certain finite factors, Proc. Japan Acad. 37 (1961), 215-216. MR 23 \#A4027.

13. R. T. Powers, Representations of uniformly hyperfinite algebras and their associated von Neumann rings, Ann. of Math. (2) 86 (1967), 138-171. MR 36 \#1989.

14. S. Sakai, On topological properties of $W^{*}$-algebras, Proc. Japan Acad. 33 (1957), 439-444. MR 20 \#5437.

15. I. E. Segal, Tensor algebras over Hilbert spaces. II, Ann. of Math. (2) 63 (1956), 160-175. MR 17, 1114.

16. E. Størmer, On anti-automorphisms of von Neumann algebras, Pacific J. Math. 21 (1967), 349-370. MR 35 \#3455.

17. —, The even CAR-algebra, Comm. Math. Phys. 16 (1970), 136-137.

Massachusetts Institute of Technology,

Cambridge, Massachusfetts 02139 